\title{
The effect of boundary layer fluctuations on the streamwise vortex structure in simulated plane turbulent mixing layers
}

\author{
W. A. McMullan* \\ Department of Engineering, University of Leicester, University Road, Leicester, LE1 \\ rRH, United Kingdom
}

\begin{abstract}
This paper details the influence of the magnitude of imposed inflow fluctuations on Large Eddy Simulations of a spatially developing turbulent mixing layer originating from laminar boundary layers. The fluctuations are physically-correlated and produced by an inflow generation technique. The imposed high-speed side boundary layer fluctuation magnitude is varied from a low-level, up to a magnitude sufficiently high that the boundary layer can be considered, in a mean sense, as nominally laminar. Cross-plane flow visualisation shows that each simulation contains streamwise vortices in the laminar and turbulent regions of the mixing layer. Statistical analysis of the secondary shear stress reveals that mixing layers originating from boundary layers with low-level fluctuations contain a spatially stationary streamwise structure. Increasing the high-speed side boundary layer fluctuation magnitude leads to a weakening of this stationary streamwise structure, or its removal from the flow entirely. The mixing layer growth rate reduces with increasing initial fluctuation level. These findings are discussed in terms of the available experimental data on mixing layers, and recommendations for both future experimental and numerical research into the mixing layer are made.
\end{abstract}

Keywords: Large Eddy Simulation, Streamwise Vortices, Mixing Layers

\footnotetext{
*Corresponding author: andrew.mcmullan@le.ac.uk
} 


\section{Introduction}

The use of numerical simulation techniques such as Direct Numerical Simulation (DNS) and Large Eddy Simulation (LES) to compute turbulent flows is becoming more widespread thanks to ever increasing computing power. Extremely well-resolved simulations of turbulent flows to reasonably high Reynolds numbers are now attainable. For the spatially-developing mixing layer flow, it is expected that these simulation methods will assist in solving the outstanding problems that persist in the field, in spite of seventy years of extensive research. Over this period of time, the mixing layer that forms between two merging parallel streams of fluid has proven to be a remarkably challenging flow configuration. A large spread in the growth rates of mixing layers has been reported, as reviewed by Yoder et al. [1]. Explanations for the discrepancies in observed growth rates include the laminar or turbulent state of the separating high-speed side boundary layer [2,3], and even whether the measured flows could be considered as truly fully-developed [4]. Regardless of the root cause, the above investigations show that the mixing layer displays a hypersensitivity to its initial conditions, and their effects persist to Reynolds numbers (based on the mixing layer visual thickness and velocity difference across it) that are in excess of those found in flows of practical engineering interest.

The presence of organised structures in the plane turbulent mixing layer has been acknowledged for over forty years. Large-scale, spanwise-orientated structures were observed in contemporary studies of the low Reynolds number flow [5], and its high Reynolds number counterpart [6]. Since their discovery, these large-scale coherent structures have been the subject of intensive study, both experimentally [7] - [10], and computationally [11] - [19]. Growth and entrainment models of the mixing layer have been developed from these observations $[19,20]$, but doubt remains as to whether such structures are ubiquitous for non-idealised initial conditions [21].

In addition to the spanwise structure a secondary, streamwise-orientated vortex structure has been observed in the mixing layer. Discovered through flow visualisation as 'streaks' [7], subsequent experiments revealed that the streaks are manifestations of a statistically-stationary streamwise vortex structure [10]. First postulated as an unstable response of the layer to threedimensional perturbations in the flow upstream of the mixing layer [22], it was later shown that the formation of streamwise vortices is linked to residual streamwise vortices in the upstream separating high-speed side bound- 
ary layer [23]. The evolution of these streamwise vortices does appear to be somewhat facility dependent. It has been observed that changes in the flow smoothing screens upstream of the mixing layer can affect the spanwise locations of the streamwise vortices [10], and that small surface imperfections in the splitter plate can provide anchor points for them [24]. Further experiments have shown that small changes in the initial conditions caused by switching the freestreams between legs in a wind tunnel can produce measurable differences in the streamwise vortex properties [25]. In some experiments the streamwise structure spacing changed in a stepwise fashion, in conjunction with the interactions that occurred between the primary spanwise structures $[7,24,26]$. Other experiments, however, have shown no evidence of changes in streamwise structure spacing with increasing streamwise distance $[22,27]$. It is not clear if a spatially stationary streamwise structure is ubiquitous for all initial conditions, as a two-stream mixing layer that originated from a turbulent high-speed side boundary layer did not contain a spatially stationary streamwise structure [28].

Eddy-resolving simulations of spatially-developing mixing layers should be able to capture the features described above. There are some examples of DNS studies in the literature [16], [17], with reasonably high Reynolds numbers attained. Large Eddy Simulation is an attractive numerical method for the simulation of mixing layers, as the large-scale structures which dictate the flow evolution are explicitly resolved by LES. Published research into the use of LES for mixing layer simulations has largely focused on idealised flows, rather than direct comparisons with reference experimental data [16], [17], [29]. In these studies the flow originates from what can be considered as idealised inflow conditions (in this paper the terms 'initial conditions' and 'inflow conditions' are used interchangably), where a base mean velocity profile is perturbed through a white noise disturbance field. Where simulations with this type of inflow condition have been performed against a reference experiment, reasonable agreement with the mean flow statistics has been obtained [18] [19]. The continuous linear growth mechanism, reported in experiments [4], has also been captured in numerical simulation [19]. In spatially developing mixing layer simulations originating from a white-noise disturbance environment, evidence for a spatially developing streamwise structure is lacking $[29,30]$.

Numerical white noise is, of course, not a physically realistic representation of the fluctuations found in real flows. In order to produce inflow fluctuations that are both spatially- and temporally-correlated, an inflow gen- 
eration technique must be used. Application of an inflow generation method to produce physically-correlated, low-level fluctuations in the upstream laminar boundary layers of a mixing layer simulation has resulted in a mixing layer that contained a spatially stationary streamwise vortex structure [30]. The statistical properties of this streamwise structure compared extremely well with experimental data [31]. The streamwise structure originated from residual streamwise vorticity contained in the upstream boundary layer, and the structure evolved in a stepwise fashion with downstream distance in the mixing layer. The growth rate of the mixing layer originating from these physically correlated initial conditions was also observed to increase by $15 \%$ when compared to a simulation originating from a white noise fluctuation environment of the same disturbance magnitude; the physically correlated simulation producing better agreement with reference data.

In order to produce an accurate numerical simulation of any mixing layer experiment, detailed information on the initial conditions of the flow is essential. This includes the mean streamwise velocity profile of each boundary layer, and the associated velocity fluctuations for all three velocity components. This complete set of mixing layer initial conditions has not been recorded for any experimental study available in the literature. Where boundary layer velocity fluctuation data has been recorded, only the streamwise component has been reported [32], [33]. All reported simulations of plane mixing layers are therefore a representation of the real conditions, and it is not clear how the magnitude of the imposed fluctuations influences the development of the mixing layer. The simulation studies of McMullan \& Garrett [31] have shown that low-level, physically-correlated boundary layer fluctuations produced a spatially stationary streamwise structure. That study, however, only considered one particular set of boundary layer fluctuation profiles; the connection between the magnitude of the fluctuations in the upstream separating boundary layers, and the formation and evolution of the streamwise vortices, has not been fully established.

The aim of this study is to quantify the effects of the magnitude of the upstream boundary layer fluctuations on the streamwise structure in simulations of the spatially developing mixing layer. The initially-laminar experiments of Browand and Latigo [32] provide the reference data for this study. An inflow generation technique is used to provide these physically-correlated inflow conditions. Three distinct simulations are performed, where the fluctuations in the high-speed boundary layer are successively increased. These simulations represent a range of initial fluctuations from being 'clean' (or 
two-dimensional in the mean), up to where the initial conditions are, in a statistical sense, highly three-dimensional (or nominally laminar). To maintain consistency all three simulations do, however, originate from the same mean inflow velocity profiles. The effects of the initial conditions on the simulated mixing layers will be assessed through mean flow statistics, flow visualisation, and cross-plane samples of the flow field at several streamwise locations. Section 2 provides a brief description of the research code. A brief summary of the reference experiment, and the simulation set up are described in Section 3. The results obtained from the simulations are presented in Section 4. A discussion of the results and their significance is provided in Section 5, and concluding remarks are drawn in Section 6.

\section{Numerical Methods}

A brief description of the research code is provided here; a more complete description can be found in a previous study [18]. The code solves the spatially-filtered equations for conservation of mass and momentum for a uniform density flow. A finite volume method is employed, with the primitive variables discretised using a staggered cell approach. The momentum equation is solved using a second-order, central differencing method. Time advancement is performed by the second-order accurate Adams-Bashforth method. The pressure equation is solved by a multi-grid method which ameliorates the convergence of the equation solution. The outflow condition is the commonly-used standard convective outflow condition [34]. The unresolved scales of motion are modelled using the WALE model [35]. A previous study has shown that this choice of subgrid-scale model is advantageous over the standard Smagorinsky, owing to the fact that the WALE model predicts zero eddy viscosity in the presence of pure shear [19].

A filtered passive scalar transport equation is solved using a secondorder accurate upwinding scheme, with time advancement achieved using the Adams-Bashforth method. In order to close this transport equation the standard gradient-diffusion model is employed, with the turbulent Schmidt number for the subgrid scalar flux assigned a value of $\mathrm{Sc}_{t}=0.3$. In this study the primary function of the filtered passive scalar, $\bar{\xi}$, is to act as a means of flow visualisation.

As the focus of this study is to assess the effect of the magnitude of the physically-correlated fluctuations in the initial conditions, an inflow generation technique is required. The method of Xiao et al. provides the inflow 
condition for the simulations presented here [36]. A brief summary of this method is provided here. Small inflow generation domains (commonly called 'virtual domains') are placed upstream of the main computational domain. At simulation start-up these virtual domains are initialised with the mean inflow boundary layer profile, onto which white noise fluctuations of magnitude $0.1 U$ are superimposed, where $U$ is the freestream velocity. At every subsequent time step an entire cross-stream plane just upstream of the end of the virtual domain is recycled onto its inlet plane. In order to maintain control of the flow in the virtual domains, the velocity field within the virtual domain rescaled to produce a desired set of velocity statistics at specified intervals.

\section{Simulation Setup}

\subsection{Reference Experiment}

The reference data for this study are those of the initially-laminar mixing layer experiment of Browand \& Latigo [32]. The recorded bulk flow properties of the experiment are shown in Table 1 . The quantities $\theta_{1}$ and $\theta_{2}$ are the high- and low-speed side boundary layer momentum thicknesses respectively, $U_{1}$ and $U_{2}$ are the freestream velocities of the high- and low-speed side respectively, and the velocity ratio parameter, $R=\left(U_{1}-U_{2}\right) /\left(U_{1}+U_{2}\right)$. Measurements showed that the boundary layers departing from the splitter plate were laminar in both streams, with mean velocity profiles close to Blasius form. The streamwise velocity fluctuation profiles in each stream were also recorded, but were subject to a $1 \%$ measurement error. No information on the vertical or spanwise velocity fields in the separating boundary layers was obtained. The test facility had fixed horizontal guide walls, which resulted in a small adverse pressure gradient in the test section. The large vertical extent of the test section did, however, help to minimise the impact of this pressure gradient.

\subsection{Computational Details}

The mixing layers are a simulated in a computational domain that extends $1630 \times 1326 \times 392 \theta_{i}$, which is discretised into $768 \times 256 \times 256$ cells. The parameter $\theta_{i}$ is the initial momentum thickness of the flow; the reference experiment determined that this quantity is equivalent to the high-speed side boundary layer momentum thickness, $\theta_{1}$, and this relationship is assumed here. This domain begins at the trailing edge of the splitter plate, which is 
assumed to have a negligible thickness. The grid is refined near the plane of the splitter plate such that $\Delta x_{\min } / \theta_{i}=0.46, \Delta y_{\min } / \theta_{i}=0.086$. This leads to the high-speed boundary layer being resolved with 36 points, and the lowspeed boundary layer being resolved with 48 points. The grid is stretched in the vertical direction such that the cell count is substantially reduced far from the plane of the splitter plate. The grid points in the spanwise direction are uniformly distributed. The upper and lower boundaries of the domain are modelled as slip walls, which results in an adverse pressure gradient in the flow similar to that observed experimentally [37]. The spanwise boundaries are periodic in nature.

The inflow condition for each simulation is obtained using an inflow generator that produces physically-correlated, time-dependent inflow data [36]. This method requires small computational domains upstream of the main mixing layer domain in which to generate the inflow condition. The grid resolution in these inflow generator domains matches that in the initial region of the mixing layer grid. In each stream these domains have an extent of 112 $\times 663 \times 392 \theta_{i}$, and are discretised into $256 \times 128 \times 256$ cells. The splitter plate is modelled as a solid wall of negligible thickness. In these generator domains the flow is recycled from a plane $10 \theta_{i}$ upstream of the trailing edge of the splitter plate onto the inflow plane at every time step. At intervals the flow is rescaled to the desired set of flow statistics. Slip wall boundary conditions are employed on the upper and lower guidewalls, and the spanwise boundaries are also periodic.

Three distinct simulations are performed in this study, in which the magnitude of the fluctuations in the high-speed boundary layer are successively increased. The imposed inflow conditions are shown in Figure 1. Each simulation has a mean streamwise velocity profile that closely approximates the experimental data, as shown in Figure 1a. Case RRM-L (Low-level fluctuations) has a high-speed boundary layer root mean squared (rms) fluctuation environment (Figure 1b) that can be considered representative of the conditions that were present in the reference experiment. In Case RRM-M (Medium-level fluctuations) higher levels of three-dimensional perturbations are present in the high-speed boundary layer (Figure 1c), and the boundary layer can be considered to be in an unsteady laminar state. In Case RRM-H (High-level fluctuations) elevated levels of disturbances are present in the high-speed side boundary layer (Figure 1d), and this renders the high-speed boundary layer as nominally laminar. Boundary layer fluctuation profiles similar to those used here have been recorded in experiments of mixing lay- 
ers [2], jet flows [38], and in numerical simulations of jet shear layers [39]. In all three simulations presented here the low-speed stream boundary layer has mean and fluctuating profiles corresponding to the data presented in Figures 1a-b.

For all simulations the WALE subgrid-scale model coefficient is set to $C_{w}=0.56$. A previous study has shown that this model offers significant advantages over the standard Smagorinsky model [19]. The passive scalar is assigned a value of unity in the high-speed stream, and zero in the low-speed stream. The normalised time step of each simulation is $\Delta t /\left(\theta_{1} / U_{c}\right)=0.0205$, where $U_{c}=0.5\left(U_{1}+U_{2}\right)$ is the convection velocity of the flow. Statistical samples are accumulated over a period of 20 convective flow through times. Where appropriate, the statistical sample size is further increased through spanwise averaging of the flow statistics - previous experimental work has shown that the presence of spatially stationary streamwise vortices can significantly influence single-plane flow statistics, and that spanwise-averaging of flow data leads to more representative statistics [40].

The current computational domain, and mesh resolution, have been extensively validated in previous studies. It has been shown that the choice of subgrid scale model produces vanishingly small eddy viscosity in the laminar region, and that the chosen value of $C_{w}$ has little effect on the bulk flow evolution [19]. It has also been demonstrated that the spanwise domain extent is sufficiently wide to avoid artificial confinement of the spanwise wavelength of the mixing layer $[41,42]$. A counterpart study has shown that a simulation of the initially laminar mixing layer with low-level, physically-correlated boundary layer fluctuations, produces a spatially stationary streamwise structure on the mesh used here [31].

\section{Results}

\subsection{Mean Flow}

Normalised mean streamwise velocity profiles, obtained at $x / \theta_{i}=1000$, are shown in Figure 2a. Owing to the adverse pressure gradient that exists in the computational domain, the normalisation parameter $\Delta U=\left(U_{1}-U_{2}\right)$ is based on local values of the freestream velocity. The velocity profiles shown in Figure 2a are extracted from the self-similar region of each simulated mixing layer, and all three profiles agree well with the reference data. The normalised r.m.s. streamwise velocity profile at the same streamwise location is shown in Figure 2b. All three simulations show reasonable agreement with the 
reference data, although Case RRM-L slightly over-predicts the magnitude of the fluctuations towards the outer edges of the mixing layer. The evolution of the maximum streamwise r.m.s. fluctuation as a function of streamwise distance is shown in Figure 3. The initial evolution of each simulation is markedly different. The peaks in the profiles of Cases RRM-L and RRM$\mathrm{M}$ in the region of $x / \theta_{i}<250$ are indicative of the roll-up of the flow into primary vortices, and pairing interactions that occur between them. These well-defined peaks are absent from Case RRM-H, where the fluctuation rises to a maximum at $x / \theta_{i} \approx 200$, before relaxing towards a self-similar value in the turbulent region. All three simulations produce a self-similar value of $u_{r m s}$ that is close to the bounds of experimental error, but each simulation attains a different asymptotic value of this quantity. This indicates that each simulation attains a different self-similar state.

Power spectral density plots of the streamwise velocity fluctuation along the streamwise direction in plane of the splitter plate $\left(y / \theta_{i}=0\right)$ are recorded in each simulation. Spectra recorded at particular streamwise locations are shown in Figure 4. In Case RRM-L (Figure 4) the spectra at $x / \theta_{i}=45$ shows a peak at $\mathrm{St} \approx 0.024$, which is in good agreement with the dominant instability frequency predicted by linear stability theory for a two-stream shear layer [43]. Further downstream the roll-off in the spectra approaches the $-5 / 3$ slope indicative of fully-developed turbulence. The transition to turbulence is precipitated by interactions between the primary spanwise rollers, in the presence of the secondary streamwise structure [26]. The spectrum recorded at $x / \theta_{i}=45$ in Case RRM-M (Figure $4 \mathrm{~b}$ ) shows a broader peak around $\mathrm{St} \approx 0.0205$, implying that there is a variation in the wavelength of the initial roll-up in the mixing layer. Further downstream a $-5 / 3$ roll-off is approached in the spectra, demonstrating that this flow undergoes a transition to turbulence. The transition to turbulence in this simulation occurs in the same manner as that described above. In Case RRM-H, however, a peak is not present in the spectrum at $x / \theta_{i}=45$, and there is instead an approach to a $-5 / 3$ roll-off, indicating the presence of a turbulent flow. The highly disturbed upstream high-speed boundary layer leads to the immediate formation of small-scale turbulent motions in the mixing layer of Case RRM-H, which persist throughout the streamwise extent of the simulated mixing layer.

The visual thickness of the mixing layer can be determined from the superposition of many spanwise-average passive scalar flow images. With the upper and lower edges of the mixing layer defined by spanwise-averaged scalar 
values of $\langle\bar{\xi}\rangle_{z}=0.01,0.99$ respectively, a measure of the visual thickness analogous to the $1 \%$ thickness of the mixing layer [44] is obtained. The visual growth of a turbulent plane mixing layer is expected to follow the relationship

$$
\frac{\delta_{v i z}}{x-x_{0}}=k_{v} R
$$

where $x_{0}$ is the virtual origin, and $k_{v}$ is a constant. The computed values of $k_{v}$ from each simulation are shown in Table 2 , and it is evident that $k_{v}$ is not universal. The growth rate reduces by approximately $14 \%$ between cases RRM-L and RRM-H, and there is a clear trend of a reduction in mixing layer growth rate with increasing high-speed side boundary layer fluctuation level. This trend of reduction in growth rate with elevated levels of boundary layer fluctuations in a two-stream mixing layer has been observed experimentally [33]. The computed growth rates are within the range of values reported experimentally [1].

An alternative measure of the integral thickness of the mixing layer can be obtained from the momentum thickness, defined as

$$
\theta=\frac{1}{\Delta U^{2}} \int_{-\infty}^{\infty}\left(U_{1}-\left\langle\bar{u}_{t}\right\rangle_{z}\right)\left(\left\langle\bar{u}_{t}\right\rangle_{z}-U_{2}\right) d y
$$

and is not a momentum deficit in the usual sense. The normalised momentum thickness distributions from each simulation are shown in Figure 5, along with the reference experimental data. The tailing-off of the momentum thickness curves is typical of simulations of shear flows [19, 39], and is caused by the passage of continuously growing coherent structures through the outflow plane. Each simulation produces reasonable agreement with the experimental momentum thickness distirbution, with Case RRM-L offering the closest agreement with the reference data. There are, however, clear differences in the evolution of the momentum thickness in each case. The self-similar region of the mixing layer is characterised by a linear slope in the momentum thickness distribution. The streamwise location at which a linear gradient is achieved differs in each simulation. Case RRM-L attains similarity at $x / \theta_{i} \approx 350$, Case RRM-M achieves similarity at $x / \theta_{i} \approx 450$, and Case RRM-H attains similarity at $x / \theta_{i} \approx 600$. In addition, the gradient of the linear slope differs between the simulations. The growth rate $d \theta / d x$, is computed for each simulation to be 0.02376 for RRM-L, 0.022 for RRM-H, and 0.0204 for RRM-H. Case RRM-L offers the best agreement with 
the experimental value, when $R$ is based on the inflow values [32]. For Case RRM-M the momentum thickness growth rate is under-predicted by $8 \%$, and for Case RRM-H it is under-predicted by $14 \%$.

When considered individually, the flow statistics based on the mean velocity field would lead to the conclusion that all of the three simulations performed in this study are a good representation of the experiment. The disparity in the spectra in Figure 4, and the computed rms velocity statistics of Figures $2 \mathrm{~b}-\mathrm{c}$, demonstrate that differences in the flow evolution do exist between the simulations, and that each simulation attains a different selfsimilar state. These statistics indicate that the magnitude of the high-speed stream boundary layer fluctuations have a definite influence on the evolution of the mixing layer flow. The effect that these changes in inflow conditions have on the vortex structure in the mixing layer, with a particular emphasis on the streamwise vortex structure, is outlined below.

\subsection{Flow Visualisation}

Large-scale coherent structures were first observed in the mixing layer using schlieren and shadowgraph imagery. These techniques produce averaged representations of the flow along the direction of interrogation of the optical beam. The uniform density nature of the present calculations prevents direct use of numerical schlieren but it has been shown that the passive scalar can be used to visualise structures in the mixing layer [19], and this approach is adopted here.

Figures $6 \mathrm{a}-\mathrm{c}$ show instantaneous, spanwise-averaged passive scalar maps from Cases RRM-L, RRM-M, and RRM-H respectively. These images are typical of those observed throughout each calculation. It is apparent that each simulation contains coherent vortex structures that persist along the entire streamwise extent of the computational domain. Whilst these images share similar features, there are some qualitative differences in the appearance of the structures in the simulations. In Case RRM-L (Figure 6a) the vortex cores are clearly visible, with tightly packed scalar lines angled to the horizontal indicating the interconnecting braid regions between the primary structures. In Cases RRM-M and RRM-H, however, the structures are somewhat less obvious - the structure cores are apparent from the 'bumps' on the upper and lower edges of the mixing layer, and the scalar lines in the interconnecting braids are much less tightly packed. As noted above the visual thickness growth rate decreases by $15 \%$ from Case RRM-L to Case RRM-H. Tracing a wedge defined by the upper and lower edges of the mixing layers 
shown in Figures $6 \mathrm{a}-\mathrm{c}$ demonstrates this change in the growth of the flow between the calculations.

Close-up images of typical pre-transition vortices obtained from each simulation are shown in Figure 7. For all three vortex structures the centre of rotation of the vortex lies in the vertical plane of $x / \theta_{i}=108$. At this streamwise location the local Reynolds number of the flow is approximately 12,000. The flow would typically be in a laminar state at this local Reynolds number, as a triggering interaction between the primary vortices has yet to occur [26]. The spanwise-averaged scalar distribution in Case RRM-L, shown in Figure 7a has the familiar 'swiss-roll' distribution typical of a laminar Kelvin-Helmholtz $(\mathrm{K}-\mathrm{H})$ vortex, where a central core of mixed fluid is surrounded by two tongues of fluid from the freestreams penetrating into the layer from the high- and low-speed side in the downstream and upstream braid regions respectively. Figure $7 \mathrm{~b}$ shows that a similar scalar distribution is present for the vortex obtained in Case RRM-M, but the tongues of fluid from the freestreams appear to mix out more rapidly than in Case RRM-L. In Case RRM-H, however, the scalar distribution is markedly different; the well-mixed core region is not present, and the scalar lines in the braids are much less tightly packed. The spectrum recorded at this streamwise location, shown in Figure 4c, indicates that Case RRM-H is turbulent, and the scalar distribution in this region is very similar to that found in the coherent structures further downstream.

The spanwise averaging of passive scalar data to produce these side-view images necessarily smears out any inherent three-dimensionality in the vortex structures. A qualitative assessment of the three-dimensional nature of the structures present in the flow can be made through recording cross-plane data at selected streamwise locations in the domain. These streamwise measurement stations are detailed in Table 3, with the locations expressed in terms of raw distance, normalised distance $x / \theta_{i}$, and the pairing parameter, $x_{i}^{*}=R x / 30 \theta_{i}[26]$. The vortices shown in Figure 7 were recorded at an instant when they convected through measurement station 2, and the corresponding $y-z$ cross-plane scalar distribution through the vertical plane of this measurement station are shown in Figure 8. The scalar distribution in the vortex core from Case RRM-L is shown in Figure 8a. There are two clear rows of mushroom-shaped features in the core of the structure, which reside on the upper and lower edges of the structure respectively. The spacing of these features is fairly regular across the span on both edges of the mixing layer. These mushroom-shaped formations are indicative of the pres- 
ence of streamwise vortices in the mixing layer. Analysis of time series of the cross-plane images from RRM-L shows that the streamwise vortices are wrapped around the primary spanwise structure in a manner matching that proposed by Bernal \& Roshko [10]. The scalar distribution in the vortex core from Case RRM-M, presented in Figure $8 \mathrm{~b}$, is qualitatively similar to that of RRM-L. Two rows of mushroom-shaped features are present, but they do not occupy the entire span; instead the spanwise region of $30 \leq z / \theta_{i} \leq$ 100 contains a single row of mushroom-like features, which is indicative of a braid region [10]. This implies that the primary spanwise vortex structure is bodily warped, and not aligned parallel to the span. Time series of the flow passing through the cross-plane shows that the streamwise vortices are not regularly spaced across the span throughout the sample. In Case RRM-H an irregular pattern of mushroom-like features is present in the vortex core, as shown in Figure 8c. The time series data shows that this streamwise vortex pattern is irregular in Case RRM-H. Regardless of the regularity, or otherwise, of the mushroom-like features in the scalar field, all three simulations show evidence for some form of streamwise structure in the near-field of the mixing layer.

The underlying three-dimensionality of typical post-transition structures are shown in Figure 9. These cross-plane scalar images are captured when the centre of rotation of a post-transition vortex structure is passing through sampling plane 5 . The local Reynolds number at this station is approximately 85,000 , and the flow is fully-turbulent at this location in each simulation. In Case RRM-L, shown in Figure 9a there are two clear rows of mushroomshaped features in the core of the structure. These features reside on the upper and lower edges of the structure respectively. The spacing of these features is fairly regular across the span on both edges of the mixing layer. These mushroom-shaped formations are indicative of the presence of streamwise vortices in the fully turbulent mixing layer. The scalar distribution in the core and braid regions of the post-transition structure in Case RRM-L are similar to that observed in its pre-transition counterpart in Figure 8a.

Qualitatively the scalar distributions shown for Cases RRM-M and RRM$\mathrm{H}$, shown in Figures 9b-c respectively, share similar features with those found in Case RRM-L; two rows of mushroom-shaped features are present, but their distribution across the span is rather irregular. All three visualisations produce cross-plane scalar images, however, that resemble the high Reynolds number cross-plane Laser Induced Fluorescence visualisations of the flow structure obtained experimentally [9], and indicate that a streamwise vortex 
structure survives into the high Reynolds number turbulent flow.

The visualisations shown above indicate that each simulation contains a vortex structure that is orientated in the streamwise direction, in addition to the primary spanwise coherent structures. No assessment of the quantitative nature of this streamwise structure can be made from these images. Numerical simulation allows simultaneous access to the scalar and velocity fields in the simulations, and hence quantitative statistical information on the streamwise structure can be obtained.

\subsection{Streamwise Vortex Structure}

At each measurement station the flow is sampled at a rate of $1.67 \mathrm{kHz}$, and up to 1560 planes are recorded in all three simulations. From these data the mean cross-plane flow field is computed at every measurement station, and higher-order statistics are obtained. Previous experimental [23] and computational [31] research has shown that cross-plane statistics can be distorted by the presence of a spatially stationary streamwise vortex structure, and that the secondary shear stress, $\overline{u^{\prime} w^{\prime}}$, can be used to infer the presence of this structure. The analysis presented here follows that of a previous study of the simulated mixing layer [30].

Contour maps of secondary shear stress $\overline{u^{\prime} w^{\prime}}$ from four measurement stations are shown in Figures 10, 11, and 12 for Cases RRM-L, RRM-M, and RRM-H respectively. The secondary shear stress is normalised by the square of the velocity difference across the layer, $\Delta U^{2}$. Note that the axes extents and contour levels differ between sub-figures. At station 1 in Case RRM-L (Figure 10a) there are clusters of secondary shear stress present in the flow. At a given spanwise location these clusters contain three regions of $\overline{u^{\prime} w^{\prime}}$ with two like-sign patches sandwiching a region of opposite sign. This pattern alternates along the spanwise direction. At station 3 (Figure 10b) these clusters have evolved into a single row of alternating sign secondary shear stress. It is straightforward to trace the structures present at station 3 back to the clusters present at station 1 , and shows that the upstream flow conditions provides anchor points for the initial streamwise vortex structure. At station 5 (Figure 10c) the number density of the structures across the span has decreased, and the spacing between them has consequently increased. The spacing of the structures continues to increase at station 6, as shown in Figure 10d. An interaction between the streamwise vortices visible at station 5 in the region of $210 \leq z / \theta_{i} \leq 300$ has completed by station 6 ; two like-signed vortices are enveloping a structure of opposite sign at station 5 , and a single 
streamwise vortex structure of the same sign as its two like-signed parents has taken their place at station 6 . These contour maps demonstrate that there exists a spatially stationary streamwise structure in Case RRM-L, and the maps shown here demonstrate a similar evolution of this quantity to that obtained experimentally [23].

The pattern of secondary shear stress observed in Case RRM-M (Figure 11a) is markedly different to that of Case RRM-L. In RRM-M there is no obvious clustering of $\overline{u^{\prime} w^{\prime}}$. At measurement station 3 (Figure 11b), however, some alternating bands of $\overline{u^{\prime} w^{\prime}}$ are visible. These bands are quite irregular, and have a peak secondary shear stress magnitudes that are approximately a factor of two lower than that found in Case RRM-L. At station 5 (Figure 11c) there is again some evidence for weakly banded secondary shear stress, but this banding is not consistent across the span. A large region of positive secondary shear stress is present in the region of $0 \leq z / \theta_{i} 160$, which subsequently disappears at station 6 (Figure 11d). The banding present at station 5 has also weakened significantly at station 6 , which implies that any stationary streamwise vortex structure that is present in Case RRM-M is extremely weak at this far downstream location. The streamwise vortex structure in Case RRM-M can therefore be considered as weakly spatially stationary.

In Case RRM-H Figure 12 shows that there is no evidence for any spatially stationary streamwise structure. The secondary shear stress is irregularly distributed at station 1, as shown in Figure 12a, and subsequently there is an absence of any regular banding in the $\overline{u^{\prime} w^{\prime}}$ contours at all other measurement stations. This lack of banding of the secondary shear stress demonstrates that the streamwise vortex structure in RRM-H is not, in a statistical sense, spatially stationary.

The presence of a spatially stationary streamwise vortex structure in the mixing layer causes bodily wrinkling and distortion of the flow. The wrinkling of the mixing layer can be quantified through plotting the mean streamwise velocity across the span in the horizontal plane of the splitter plate $(y=0)$. These profiles are plotted in Figure 13 for all three cases. The velocity profiles are normalised by the value of each profile at mid-span of the domain. The profiles from Case RRM-L are shown in Figure 13a. At station 1 there are noticeable wiggles in the velocity profile. These are caused by the transfer of weak streamwise vorticity from the upstream laminar boundary layer into the mixing layer. Further downstream a regular variation in the velocity profile is present at stations 3-7. A clear spanwise wavelength exists in 
each of these profiles, and the maximum peak-to-peak amplitude is $20 \%$. This value is comparable to other simulation data [30], and is in the range reported experimentally [23], [24]. The peaks and troughs in these profiles align with the interfaces between the streamwise vortices noted in Figure 10 - a peak is an interface between two streamwise vortices with a common upflow, and a trough is an interface between two streamwise vortices with a common downflow. This regular pattern in the velocity profiles provides further evidence of a spatially stationary streamwise structure in Case RRML, which serves to bodily wrinkle the mixing layer. The number of peaks and troughs in the profiles decreases with increasing downstream distance from station 3, which indicates that the streamwise vortex structure evolves as the flow proceeds downstream. The amplitude of the variations decreases with increasing streamwise distance at the furthest downstream station the profile is reasonably flat, but some low-amplitude peaks and troughs are still visible. This pattern of evolution is consistent with experiments [23], and previous simulation work [30].

The velocity profiles extracted from Case RRM-M have a somewhat different character to those extracted from Case RRM-L. A very large variation in the velocity profile at station 1 is present, and this is caused by the unsteady behaviour of the upstream high-speed side boundary layer. At stations 3-5 some peaks and troughs in the profiles are visible, and these also align with interfaces between the banding of alternating sign $\overline{u^{\prime} w^{\prime}}$ seen in Figure 11 . These peaks and troughs appear to be positioned at isolated locations across the span, and the maximum amplitude variation is $8 \%$ - considerably lower than that observed in Case RRM-L. The value of $8 \%$ is, however, within the range reported experimentally $[23,45]$. The amplitude of the variations also decreases with increasing streamwise distance, until the profile at station 7 is effectively flat.

The profiles extracted from Case RRM-H are effectively flat and parallel at each measurement station. These profiles demonstrate that, in a mean sense, there is little wrinkling of the mixing layer. Coupled with the secondary shear stress maps presented in Figure 12, it is clear that Case RRM-H is devoid of any statistically stationary streamwise structure, and the mean flow is effectively statistically two-dimensional.

Further evidence of the wrinkling of the mixing layer can be obtained from the spanwise variation of the mixing layer thickness. At each measurement station the mixing layer thickness is computed across the entire span, and the standard deviation of the spanwise averaged mean thickness 
is computed. The mixing layer thickness is computed from the velocity field, and is obtained from the vertical distance between the locations where $U_{0.01}=U_{2}+0.01\left(U_{1}-U_{2}\right)$, and $U_{0.99}=U_{2}+0.99\left(U_{1}-U_{2}\right)$. The streamwise evolution of the thickness variation is shown in Figure 14, with the streamwise distance presented in terms of the pairing parameter [26], to facilitate comparison with experimental data obtained from a mixing layer of comparable Reynolds number [23]. The thickness variation of each case is shown in Figure 14. The trend of Case RRM-L agrees well with the experimental data; an initially low variation rapidly increases to a maximum at station $2\left(x_{i}^{*}=2.4\right)$, and then subsequently decays to approximately $3 \%$ in the far-field. The peak variation is just downstream of the roll-up of the flow into primary vortices, which occurs on average at $x_{i}^{*} \approx 2.4$. The roll-up of the flow results in an amplification of the residual streamwise vorticity into a row of alternating sign streamwise vortices [23], and has also been observed in mixing layer simulations developing from initially laminar conditions with low-level, physically-correlated inflow fluctuations [30]. For Case RRM-M a qualitatively similar trend is observed, in that a peak in the variation is reached at stations $2-3$, downstream of which the variation decays to approximately two-dimensionality. The magnitude of the peak variation, however, is considerably lower than both the experimental data, and that of Case RRM-L. The lack of clustering of the streamwise vortex structures in the initial region of the mixing layer effectively dampens the formation of a row of alternating sign streamwise vortices following the roll-up of the mixing layer into primary spanwise vortices. The weak statistically stationary streamwise vortices that do occur in isolated regions across the span do not wrinkle the flow as significantly as those in Case RRM-L. In Case RRM-H a very low amplitude peak is observed at station 2 , but its magnitude is little larger than the far downstream variations obtained in the other two cases. At all locations Case RRM-H is effectively statistically two-dimensional, as the absence of a stationary streamwise vortex structure results in no significant deviations in the thickness across the span.

\section{Discussion}

The simulation results presented here demonstrate that both the formation and the evolution of the streamwise vortex structure in the plane mixing layer are heavily influenced by the mixing layer initial conditions. The hypersensitivity of the simulated mixing layer to the nature of the imposed 
boundary layer fluctuations has been studied elsewhere [30] - a simulation originating from laminar inflow conditions with low-level white noise disturbances fails to produce a spatially stationary streamwise vortex structure, whilst physically correlated fluctuations of the same magnitude produces a simulated mixing layer that does contain a stationary streamwise vortex structure. In the present study it has been shown that the streamwise vortex structure displays a hypersensitivity to the magnitude of the physically correlated fluctuations; low-level fluctuations produce a stationary streamwise vortex structure, whilst increasing levels of fluctuations serve to markedly alter its evolution, or to remove the stationary streamwise structure from the mixing layer entirely.

The sensitivity of the stationary streamwise structure to upstream flow conditions has been recognised in experiments. Changes in the flow screens [10], swapping the legs in which the freestreams are fed into the test section [25], and small nicks in the splitter plate [24] have all been shown to affect the spanwise locations at which streamwise vortices occur. Experiments performed months apart to the same mean flow conditions in the same rig produced no change in the spanwise locations of the streamwise structure [26]. All of the experiments described above were performed in wind tunnels where attempts were made to minimise the upstream disturbances, and it is reasonable to make the initial conditions as 'two-dimensional' as possible. Where the streamwise velocity fluctuation profile of the high-speed side boundary layer has been measured, the magnitude of the fluctuations is comparable to those imposed in RRM-L [32], [33]. Very few experiments have attempted to study the plane mixing layer originating from initially laminar conditions with high-level fluctuations [2], [33], [44], and those that have did not determine the streamwise vortex structure in the flow. The findings of this simulation study suggest that elevated inital fluctuations leads to the elimination of the spatially stationary streamwise structure from the mixing layer. Interestingly this phenomenon has been observed in experiments of the plane mixing layer originating from a turbulent high-speed side boundary layer [28]. For the initially-laminar flows considered here, it seems likely that the presence of unsteady small-scale motions in the initial region of the mixing layer prevents the process of amplification of residual upstream streamwise vorticity into alternating streamwise vortices by the braid instability once the mixing layer rolls up into primary vortices. Further experiments will need to be performed, however, to confirm this hypothesis.

The simulations presented here clearly demonstrate a dependency of the 
growth rate on the mixing layer initial conditions. Mixing layer initial conditions are typically documented by the freestream velocities, integral boundary layer parameters such as the momentum thickness, and a freestream turbulence intensity. Some studies do document the mean streamwise velocity of the upstream boundary layers, but documentation of the fluctuation profiles is exceedingly rare. By any classical measurement of the mixing layer initial conditions, all three simulations considered here originate from identical initial conditions, as outlined in Table 1. The growth rates obtained from these simulations, however, vary by up to $15 \%$, with the growth rate reducing with increasing boundary layer disturbance magnitude. The growth rates obtained from the current simulations are within the range reported experimentally [1]. In two-stream mixing layer experiments where the highspeed side boundary layer fluctuations were measured, [33, 46], this trend of reducing growth rate with increasing fluctuation level was also observed. It is postulated here that a possible explanation for the wide range of growth rates reported in the literature is that the lack of documentation of the initial conditions resulted in the influence of the initial fluctuations in the boundary layer being neglected.

The current findings have an impact on the role of numerical simulation in improving our understanding of turbulent flows. Direct Numerical Simulation is free from errors introduced by the modelling of turbulent scales of motion, but that does not mean that a DNS of the mixing layer will be unaffected by the imposed inflow condition. None of the reported DNS of the mixing layer displayed any evidence of spatially stationary streamwise vortices [16], [17], and this is owing to the fact that the initial fluctuation environment of these simulations were based on pseudo-random white noise. It can be expected that, when a physically-correlated inflow condition is employed, a DNS of the mixing layer will display the sensitivity to the imposed inflow condition as that presented here. This then raises a question as to what is the purpose of DNS? If DNS is to gain insights into 'real' turbulence, then the imposed flow conditions must accurately match the 'real' flow. As described above small changes to mixing layer initial conditions can produce changes in the streamwise vortex structure [24, 25], and hence a successful DNS of the real flow should be able to both capture and provide physical insights into so-called experimental uniqueness. This will require the use of a physically-correlated inflow, which necessarily demands adequate documentation of the initial conditions of experiments. If, on the other hand, DNS serves to provide insights into idealised flows that are free from experimen- 
tal uniqueness, it must be recognised that these idealised conditions may produce a simulated flow that contains features which are not necessarily representative of the mixing layer found in the laboratory [31].

Recent simulations have attempted to assert the effectiveness of twodimensional simulations at describing the salient physics of the laboratory mixing layer [47]. Any simulation bound to two dimensions, regardless of numerical method, is singularly incapable of capturing the streamwise vortex structure that is known to exist in the mixing layer [7, 10, 23]. Differing interpretations of the turbulent coherent structures that exist in the mixing layer have been mooted. One interpretation views the turbulent structures as quasi-two-dimensional, on top of which a secondary structure rides passively [48]. A differing interpretation views the large-scale turbulent structures as the largest in a continuous cascade of three-dimensional motions [4]. Regardless of the true dynamics of these coherent structures, the inclusion of the third dimension in order to capture the physics of the streamwise structure is essential. The simulations presented here clearly demonstrate that the streamwise structure is altered by increased levels of upstream fluctuations, and it is not clear how this effect could be captured by a two-dimensional simulation method.

\section{Conclusions}

Simulations of the plane turbulent mixing layer have shown that the predicted streamwise vortex structure is dependent on the magnitude of the fluctuations present in the upstream high-speed side boundary layer. Lowlevel fluctuations lead to a mixing layer that contains a spatially stationary streamwise structure. Increases in the magnitude of the boundary layer fluctuations leads to either a weakening of this stationary streamwise structure, or its removal from the flow entirely. The changes in the streamwise vortex structure with increasing high-speed boundary fluctuation level are accompanied by a reduction in the mixing layer growth rate of up to $15 \%$. Each simulation presented here also attains a different self-similar state, suggesting that the large spread in reported mixing layer data may be accounted for by the influence of the initial conditions. Better documentation of experimental initial conditions is required to confirm this hypothesis. 


\section{Acknowledgements}

The simulations were performed on ALICE, the University of Leicester High Performance Computing facility. The author is grateful to Dr C. M. Coats for enlightening discussions during the research.

\section{References}

[1] Yoder, D. A., DeBonis, J. R., and Georgiadis, N. J. 2015. "Modeling of turbulent free shear flows." Computers \& Fluids, Vol. 117, pp. 212-232.

[2] Batt, R.G., 1975. "Some measurements of the effect of tripping the twodimensional shear layer." AIAA Journal, Vol. 13, pp. 245-247.

[3] Slessor, M. D., Bond, C. L., and Dimotakis, P. E. 1998. "Turbulent shear-layer mixing at high Reynolds numbers: effects of inflow conditions" Journal of Fluid Mechanics, Vol. 375, pp. 115-138.

[4] D'Ovidio, A., and Coats, C. M., 2013. "Coherent-structure evolution in turbulent mixing layers. Part 1: Experimental evidence." Journal of Fluid Mechanics, Vol. 737, pp. 466-498.

[5] Winant, C. D., and Browand, F. K., 1974. "Vortex Pairing: The mechanism of turbulent mixing layer growth at moderate Reynolds numbers." Journal of Fluid. Mechanics, Vol 63, pp. 237-255.

[6] Brown, G. L., and Roshko, A., 1974. "On density effects and large structure in turbulent mixing layers." Journal of Fluid Mechanics, Vol. 64, pp. $755-816$.

[7] Konrad, J.H., 1976. "An experimental investigation of mixing in twodimensional turbulent shear flows with applications to diffusion-limited chemical reactions." PhD Thesis, California Institute of Technology.

[8] Hernan, M.A., and Jimenez, J., 1982. "Computer analysis of a highspeed film of the plane turbulent mixing layer." Journal of Fluid Mechanics, Vol. 119, pp. 323-345.

[9] Jimenez, J., Cogollos, M., and Bernal, L. P., 1985. "A perspective view of the plane mixing layer." Journal of Fluid Mechanics, Vol. 152, pp. $125-143$. 
[10] Bernal, L. P., and Roshko, A., 1986. "Streamwise vortex structure in plane mixing layers." Journal of Fluid Mechanics, Vol. 170, pp. 499525 .

[11] Moser, R. D., and Rogers, M. M., 1991. "Mixing transition and the cascade to small scales in a plane mixing layer." Physics of Fluids A, Vol. 3 pp. 1128-1134.

[12] Rogers,, M. M., and Moser, R. D., 1992. "The three-dimensional evolution of a plane mixing layer: the Kelvin-Helmholtz rollup." Journal of Fluid Mechanics, Vol. 243, pp. 183-226.

[13] Moser, R. D., and Rogers, M. M., 1993. "The three-dimensional evolution of a plane mixing layer: pairing and transition to turbulence." Journal of Fluid Mechanics, Vol. 247, pp. 275-320.

[14] Rogers, M. M., and Moser, R. D., 1994. "Direct simulation of a selfsimilar turbulent mixing layer." Physics of Fluids, Vol. 6, pp. 903-923.

[15] Balaras, E., Piomelli, U., and Wallace, J. M., 2001. "Self-similar states in turbulent mixing layers." Journal of Fluid Mechanics, Vol. 446, pp. $1-24$.

[16] Wang, Y., Tanahashi, M., and Miyauchi, T., 2007. "Coherent fine scale eddies in turbulence transition of spatially-developing mixing layer." International Journal of Heat and Fluid Flow, Vol. 28, pp. 1280-1290.

[17] Attili, A., and Bisetti, F., 2012. "Statistics and scaling of turbulence in a spatially developing mixing layer at $\mathrm{Re}_{\lambda}=250$." Physics of Fluids, Vol. 24, doi: 10.1063/1.2696302

[18] McMullan, W. A., Gao, S., and Coats, C. M., 2009. "The effect of inflow conditions on the transition to turbulence in Large Eddy Simulations of spatially developing mixing layers." International Journal of Heat and Fluid Flow, Vol. 30, pp. 1054-1066.

[19] McMullan, W. A., Gao, S., and Coats, C. M., 2015. "Organised large structure in the post-transition mixing layer. Part 2. Large eddy simulation." Journal of Fluid Mechanics, Vol. 762, pp. 302-343. 
[20] Dimotakis, P.E., 1986. "Two-dimensional shear layer entrainment." AIAA Journal, Vol. 24, pp. 1791-1796.

[21] Chandrsuda, C., Mehta, R.D., Weir, A.D., and Bradshaw, P., 1978. "Effect of free-stream turbulence on large structure in turbulent mixing layers." Journal of Fluid Mechanics, Vol. 85, pp. 693-704.

[22] Lasheras, J. C., Choi, J. S., and Maxworthy, T., 1986. "On the origin and evolution of streamwise vortical structures in a plane, free shearlayer." Journal of Fluid Mechanics, Vol. 172, pp. 231-258.

[23] Bell, J. H., and Mehta, R. D., 1992. "Measurement of streamwise vortical structures in a plane mixing layer." Journal of Fluid Mechanics, Vol. 239, pp. 213-248.

[24] Jimenez, J., 1983. "A spanwise structure in the plane shear layer." Journal of Fluid Mechanics, Vol. 132, pp. 319-336.

[25] Plesniak, M. W., Bell, J. H., and Mehta, R. D., 1993. "Effects of small changes in initial conditions on mixing layer three-dimensionality." $E x$ periments in Fluids, Vol. 14, pp. 286-288.

[26] Huang, L-S, and Ho, C-M., 1990. "Small scale transition in a plane mixing layer." Journal of Fluid Mechanics, Vol 210, pp. 475-500.

[27] Breidenthal, R., 1978. "A chemically reacting plane shear layer." $\mathrm{PhD}$ Thesis, California Institute of Technology.

[28] Bell, J.H., and Mehta, R.D., 1990. "Development of a two-stream mixing layer from tripped and untripped boundary layers." AIAA Journal, Vol. 28, pp. 2034-2042.

[29] Comte, P., Silvestrini, J. H., and Begou, P., 1998. "Streamwise vortices in large-eddy simulations of mixing layers." European Journal of Mechanics/B Fluids, Vol. 17, pp. 615-637.

[30] McMullan, W.A., and Garrett, S.J., 2016. "On streamwise vortices in large eddy simulations of initially laminar plane mixing layers." International Journal of Heat and Fluid Flow, Vol. 59, pp. 20-32. 
[31] McMullan, W.A., and Garrett, S.J., 2016 "Initial condition effects on large structure in numerical simulations of plane mixing layers." Physics of Fluids, Vol. 28, pp. 01511-1-27.

[32] Browand, F. K., and Latigo, B. O. 1979. "Growth of the two-dimensional mixing layer from a turbulent and nonturbulent boundary layer." Physics of Fluids, Vol. 22, pp. 1011-1019.

[33] Pickett, L. M., and Ghandhi, J. B., 2002. "Passive scalar mixing in a planar shear layer with laminar and turbulent inlet conditions." Physics of Fluids, Vol. 14, pp. 985-998.

[34] Voke, P., and Potamitis, S. G., 1994. "Numerical simulation of a lowReynolds-number turbulent wake behind a flat plate." International Journal of Numerical Methods in Fluids, Vol. 19, pp. 377-393.

[35] Nicoud, F., and Ducros, F., 1999. "Subgrid-scale stress modelling based on the square of the velocity gradient tensor." Flow, Turbulence and Combustion, Vol. 62, pp. 183-200.

[36] Xiao, F., Dianat, M., and McGuirk, J. J., 2014. "LES of turbulent liquid jet primary breakup in turbulent coaxial flow." International Journal of Mulitphase Flow, Vol. 60, pp. 103-118.

[37] McMullan, W.A., 2014. "On the growth of a plane mixing layer from laminar or turbulent initial conditions." AIAA Aviation, Atlanta, GA. AIAA-2014-3096.

[38] Hussain, A.K.M.F., and Zedan, M.F., 1978. "Effect of the initial condition on the axisymmetric free shear layer: effect of the initial fluctuation level." Physics of Fluids, Vol. 21, pp. 1475-1481.

[39] Bogey, C., and Bailly, C., 2010. "Influence of nozzle-exit boundary-layer conditions on the flow and acoustic fields of initially laminar jets." Journal of Fluid Mechanics, Vol. 663, pp. 507-538.

[40] Bell, J.H, Plesniak, M.W., and Mehta, R.D., 1992. "Spanwise averaging of plane mixing layer properties." AIAA Journal, Vol. 30, pp.835-837.

[41] McMullan, W.A., 2015. "Spanwise domain effects on the evolution of the plane turbulent mixing layer." International Journal of Computational Fluid Dynamics, Vol. 29, pp. 333-345. 
[42] McMullan, W.A. "Spanwise domain effects on streamwise vortices in the plane turbulent mixing layer." Under Review, European Journal Mechanics B/Fluids.

[43] Monkewitz, P. A., and Huerre, P., 1982. "Influence of velocity ration on the spatial instability of mixing layers." Physics of Fluids, Vol. 25, pp. $1137-1143$.

[44] Karasso, P.S., and Mungal, M.G., 1996. "Scalar mixing and reaction in plane liquid shear layers." Journal of Fluid Mechanics, Vol. 323, pp. $23-63$.

[45] Plesniak, M.W., and Johnston, J.P., 1989. "The effects of longitudinal curvature on turbulent two-stream mixing layers." Rep. MD-54, Department of Mechanical Engineering, Stanford University.

[46] Weisbrot, I., Einav, S., and Wygnanski, I., 1982. "The nonunique rate of spread in the two-dimensional mixing layer." Physics of Fluids, Vol. 25, pp. 1691-1693.

[47] Suryanarayanan, S., and Narasimha, R., 2017. "Insights into the growth rate of spatially evolving plane turbulent free-shear layers from 2D vortex-gas simulations." Physics of Fluids, Vol. 29, pp. 020708-16.

[48] Brown, G., and Roshko, A., 2012 "Turbulent shear layers and wakes." Journal of Turbulence, Vol. 13. pp. 1-32. 


\begin{tabular}{ccccc}
$U_{1}\left(\mathrm{~ms}^{-1}\right)$ & $\theta_{1}(\mathrm{~mm})$ & $U_{2}\left(\mathrm{~ms}^{-1}\right)$ & $\theta_{2}(\mathrm{~mm})$ & $\mathrm{R}$ \\
\hline 25.6 & 0.457 & 5.2 & 0.86 & 0.66 \\
\hline
\end{tabular}

Table 1: Flow Parameters.

\begin{tabular}{cc} 
Case & $k_{v}$ \\
\hline RRM-L & 0.366 \\
RRM-M & 0.34 \\
RRM-H & 0.32 \\
\hline
\end{tabular}

Table 2: Visual thickness growth rate constants obtained from the simulations.

\begin{tabular}{cccc} 
Station & $x(\mathrm{~m})$ & $x / \theta_{i}$ & $x_{i}^{*}$ \\
\hline 1 & 0.02 & 44 & 0.96 \\
2 & 0.05 & 108 & 2.4 \\
3 & 0.1 & 217 & 4.8 \\
4 & 0.15 & 326 & 7.2 \\
5 & 0.3 & 652 & 14.4 \\
6 & 0.45 & 978 & 21.5 \\
7 & 0.6 & 1304 & 28.8 \\
\hline
\end{tabular}

Table 3: Cross-stream measurement locations. 


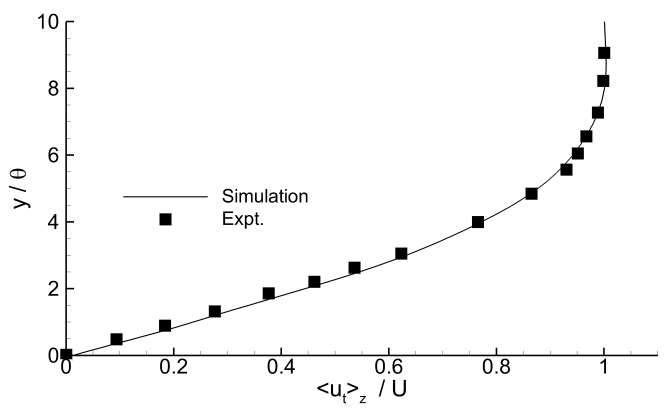

(a) Mean streamwise velocity.

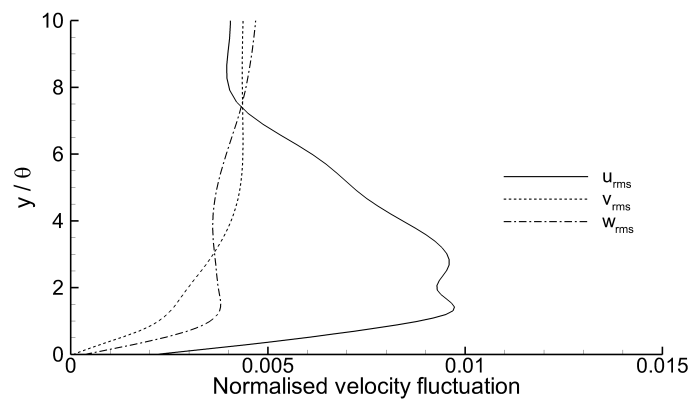

(b) RRM-L.

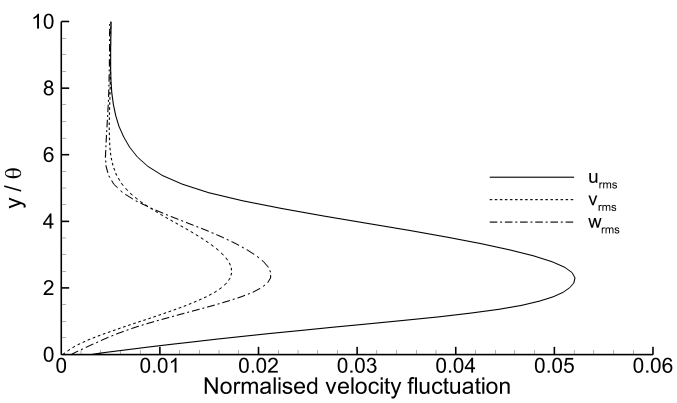

(c) RRM-M.

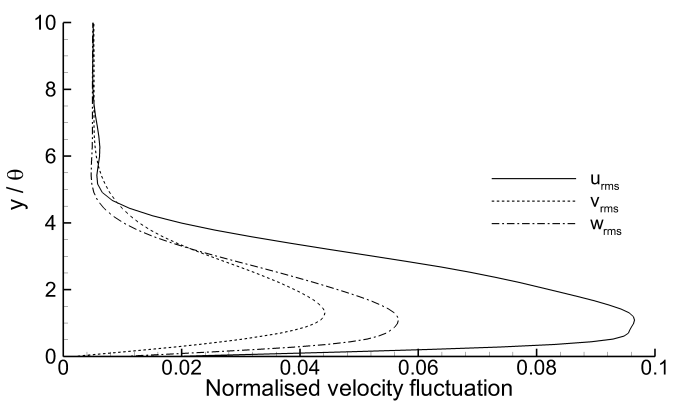

(d) RRM-H.

Figure 1: High-speed side inflowy conditions for the simulations. 


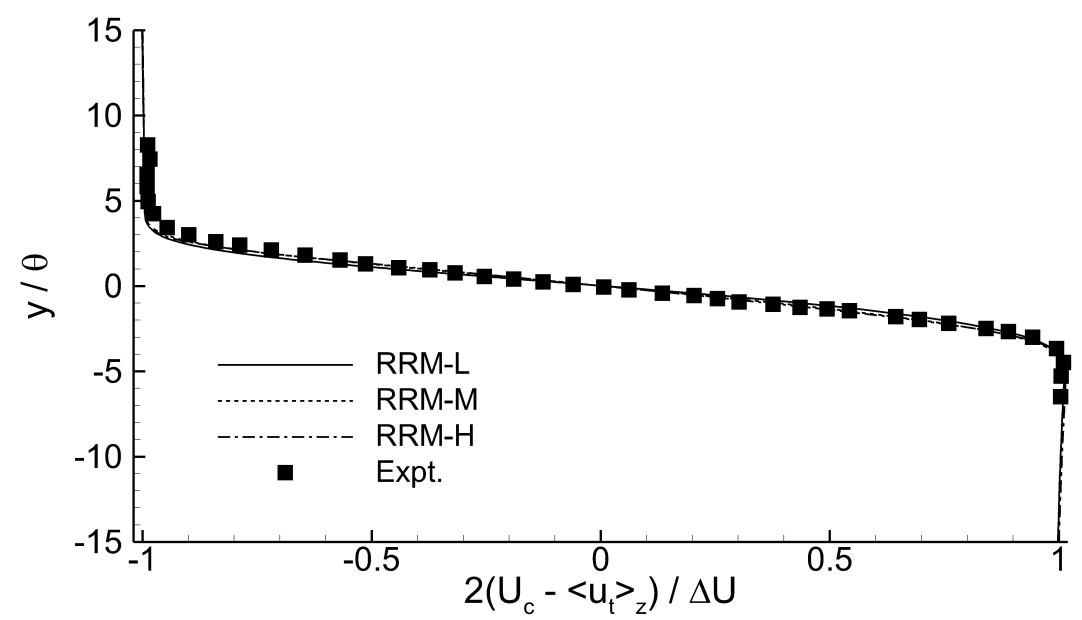

(a) Mean streamwise velocity

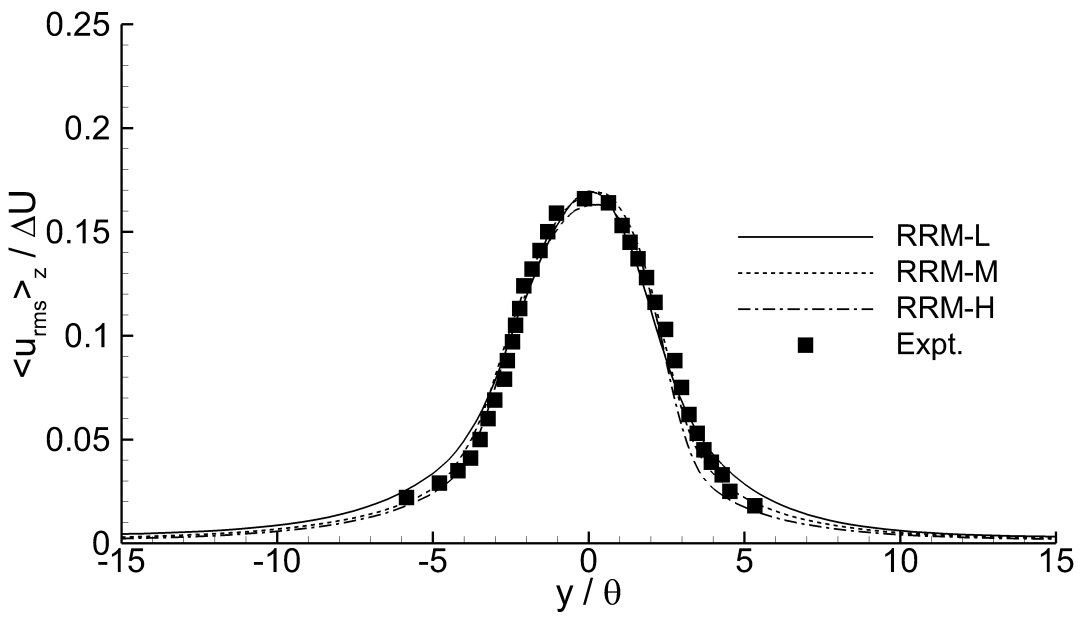

(b) Streamwise velocity fluctuation.

Figure 2: Flow statistics obtained at $x / \theta_{i}=1000$. 


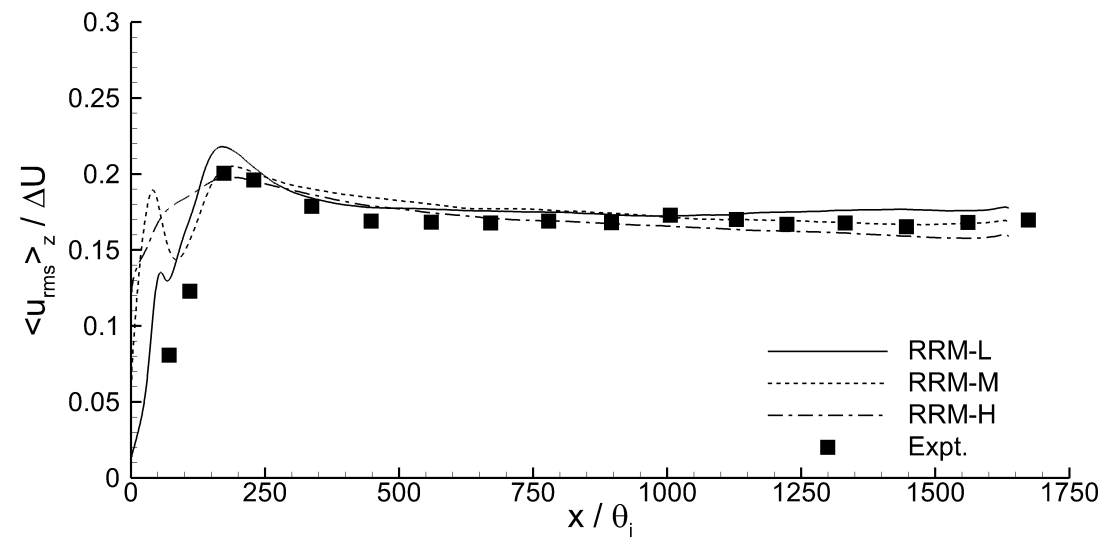

Figure 3: Variation of maximum velocity fluctuation with streamwise distance in the simulations. 


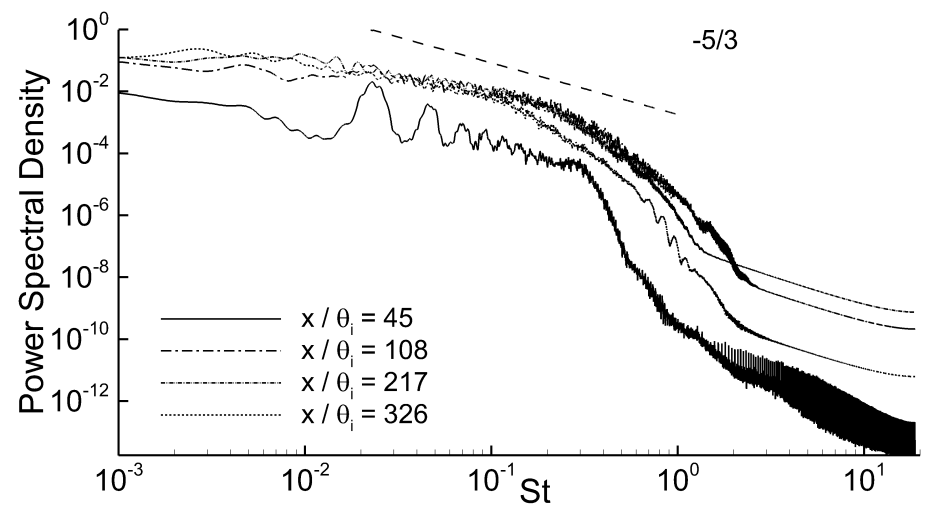

(a) RRM-L

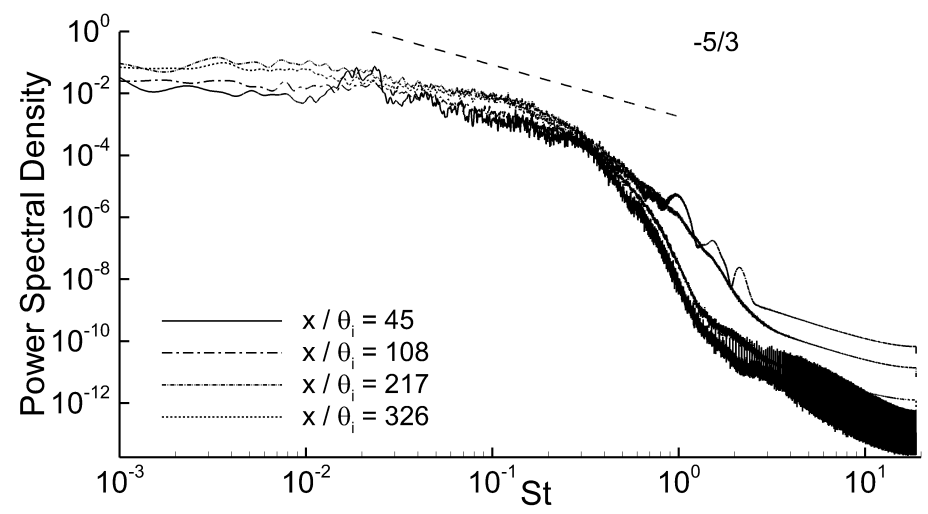

(b) RRM-M

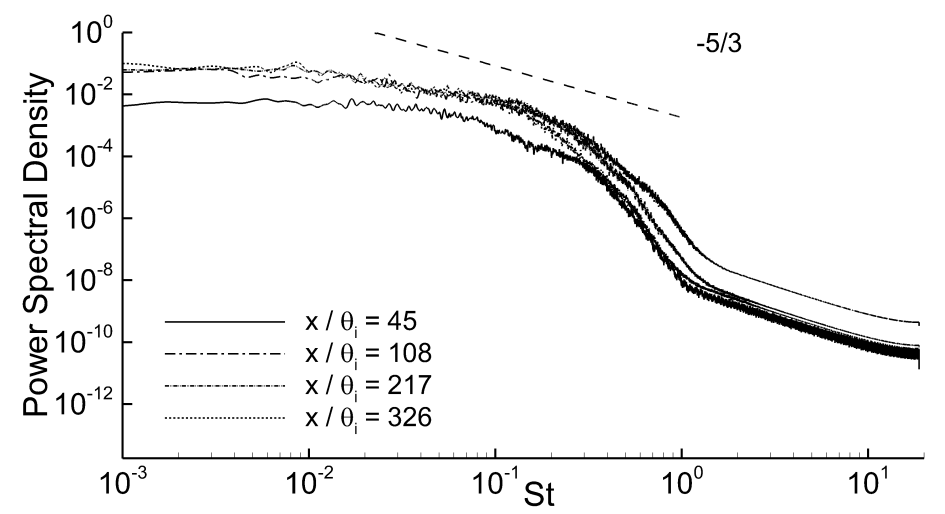

(c) RRM-H

Figure 4: Power spectral density distributions of streamwise velocity fluctuations. 


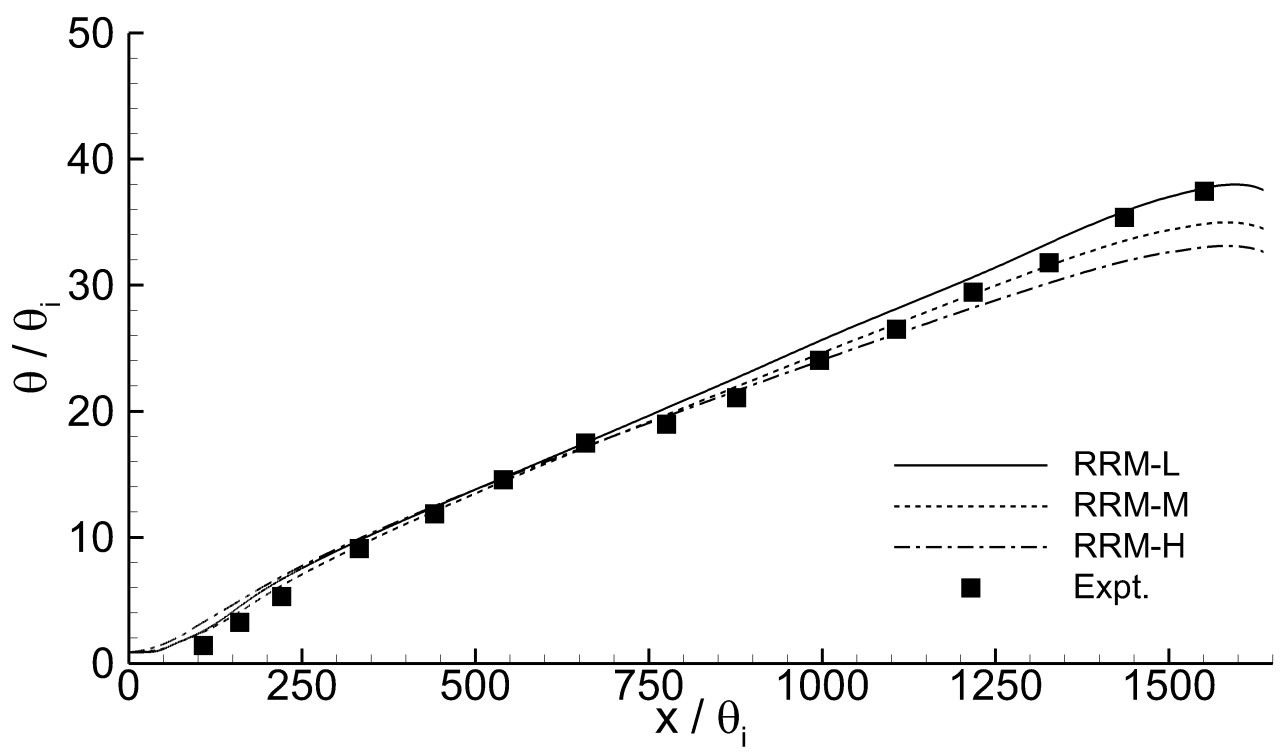

Figure 5: Normalised mixing layer momentum thickness. 


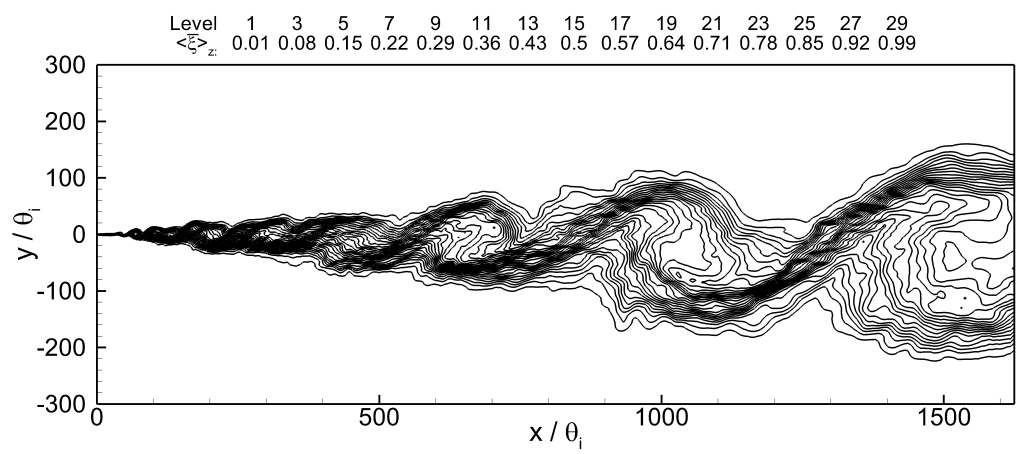

(a) RRM-L.

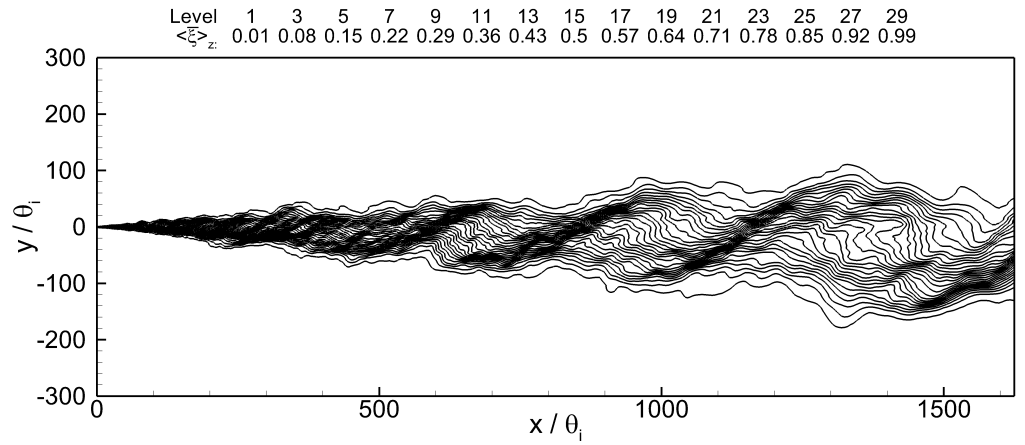

(b) RRM-M.

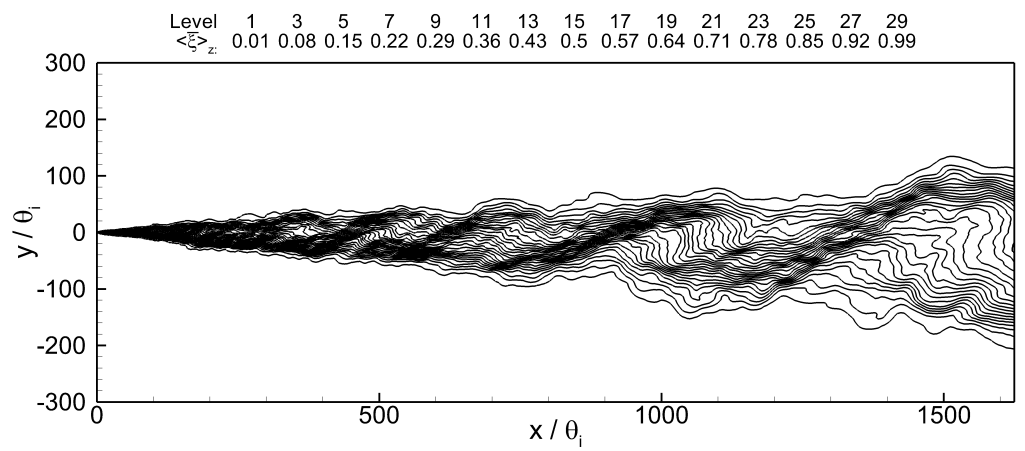

(c) RRM-H.

Figure 6: Typical instantaneous, spanwise-averaged passive scalar distributions. 


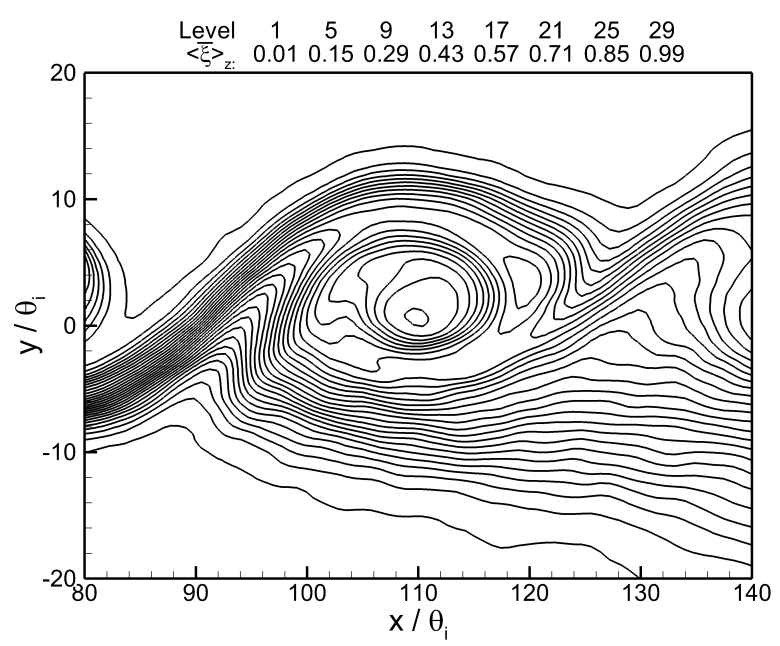

(a) RRM-L.

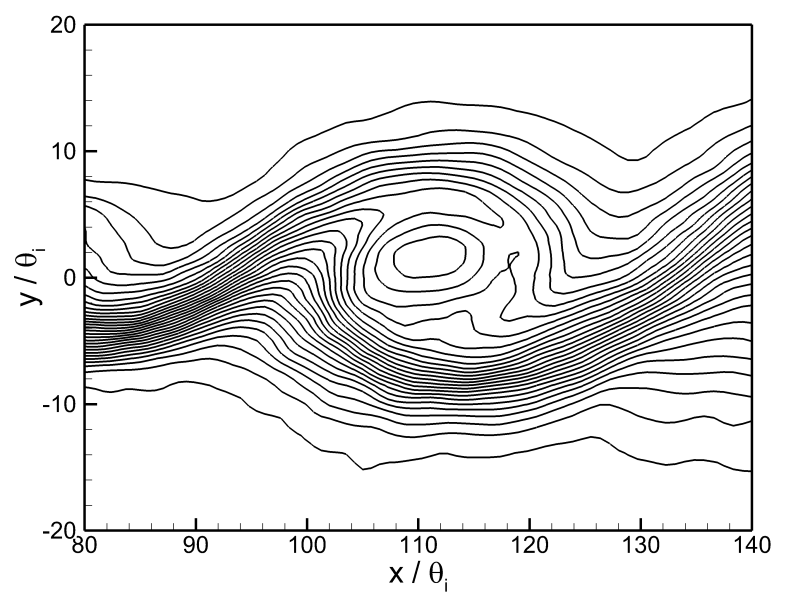

(b) RRM-M.

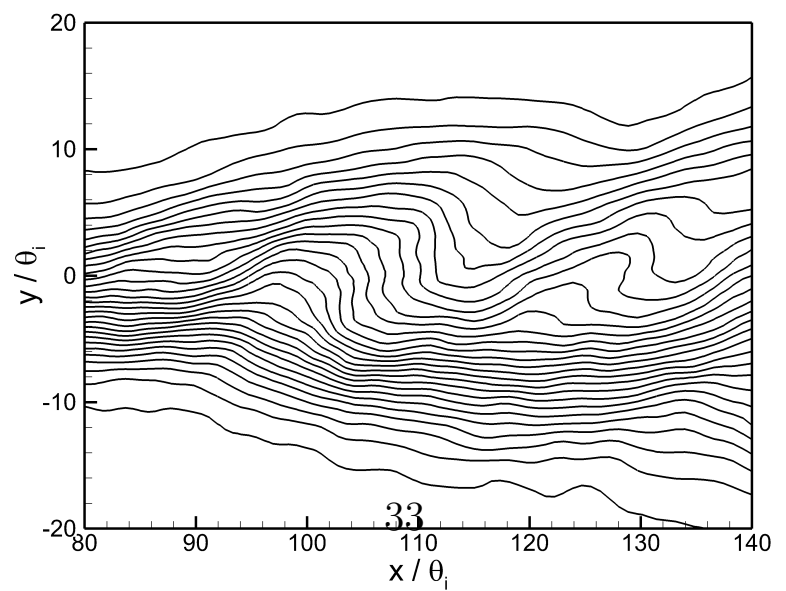

(c) RRM-H.

Figure 7: Typical vortex structures passing through measurement station 2. 


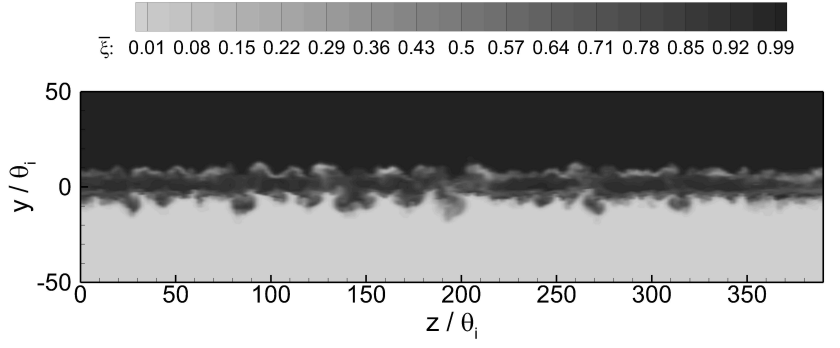

(a) RRM-L.

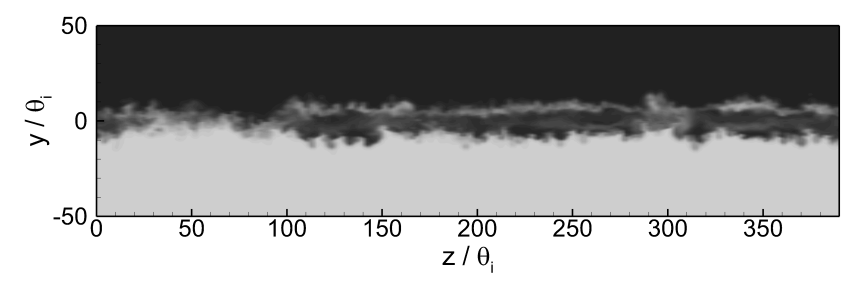

(b) RRM-M.

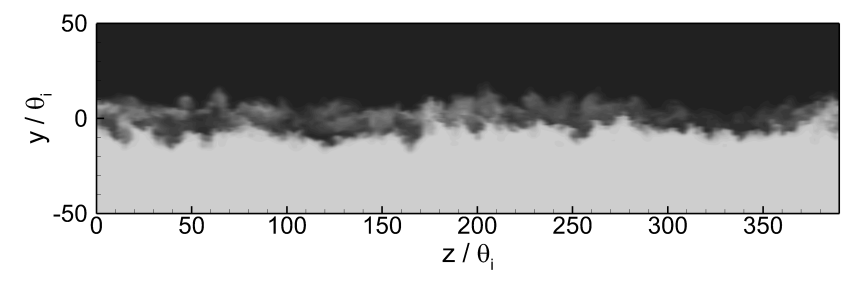

(c) RRM-H.

Figure 8: Instantaneous cross-plane scalar distributions through the cores of the vortex structures shown in Figure 7. 


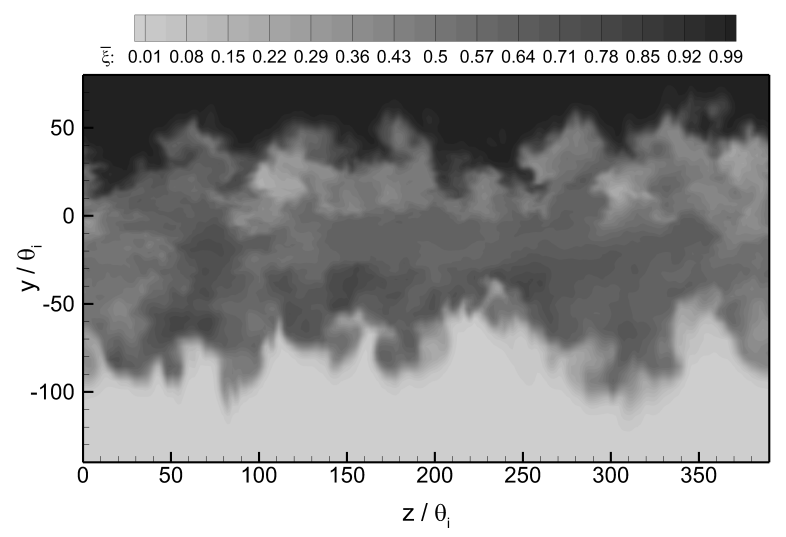

(a) RRM-L.

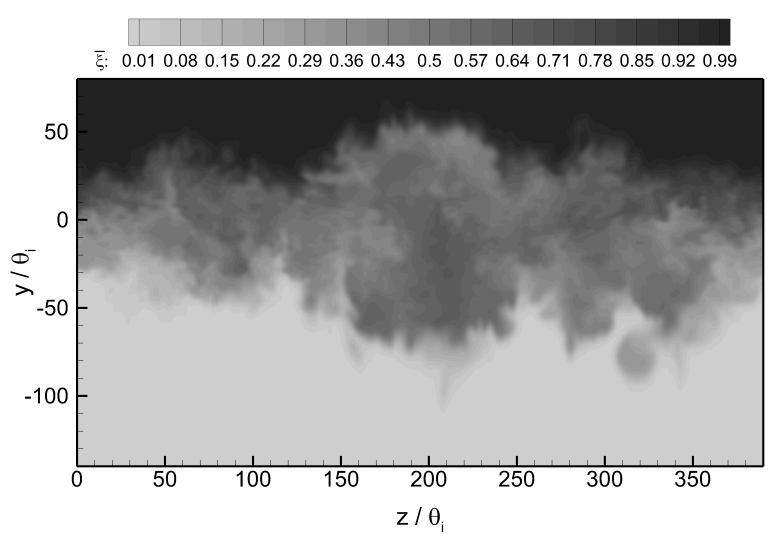

(b) RRM-M.

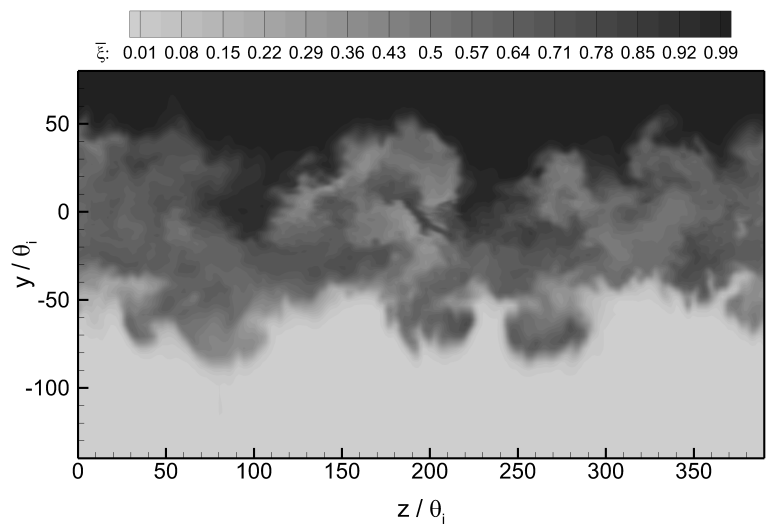

(c) RRM-H.

Figure 9: Instantaneous cross-plane scalar distributions, recorded at an instant when a structure core passes through measurement station 5 . 


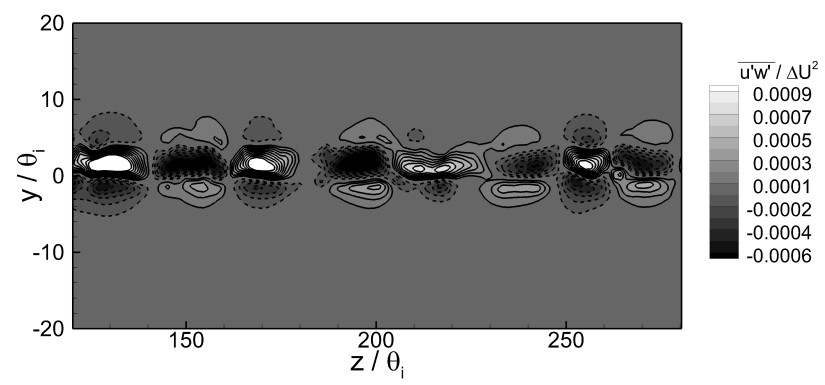

(a) Station 1 .

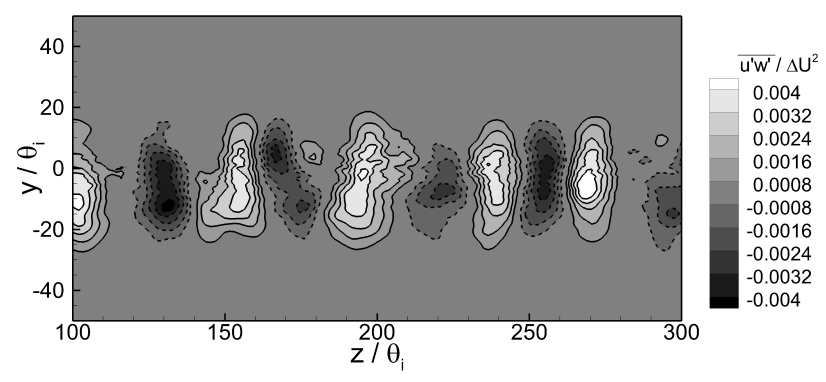

(b) Station 3 .

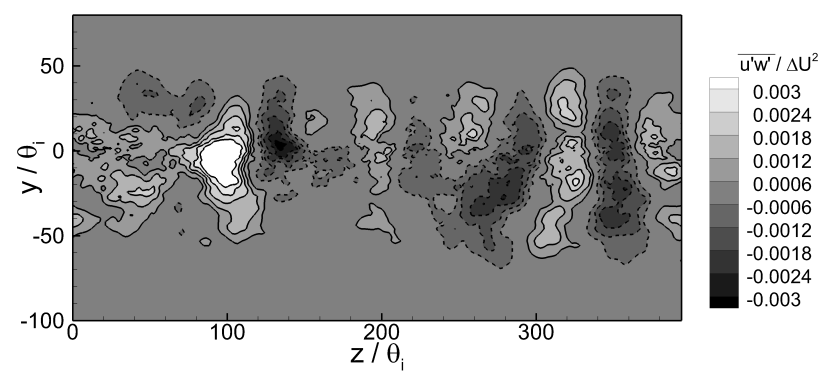

(c) Station 5 .

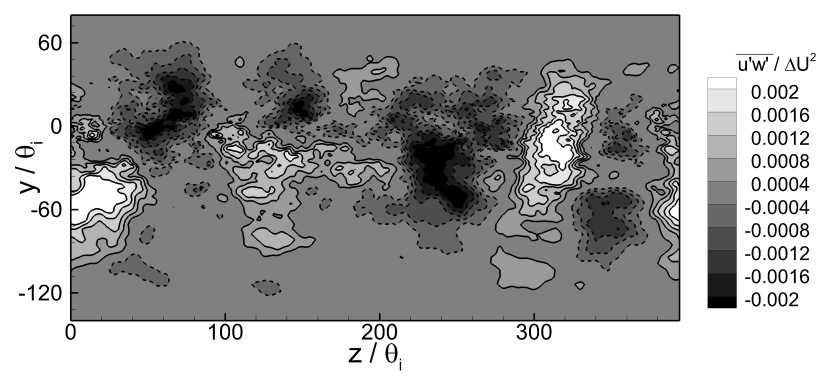

(d) Station 6 .

Figure 10: Cross-plane mean secondary shear stress contours in Case RRM-L. Note the changes in axes extent, and changes in contodur levels, between images. 


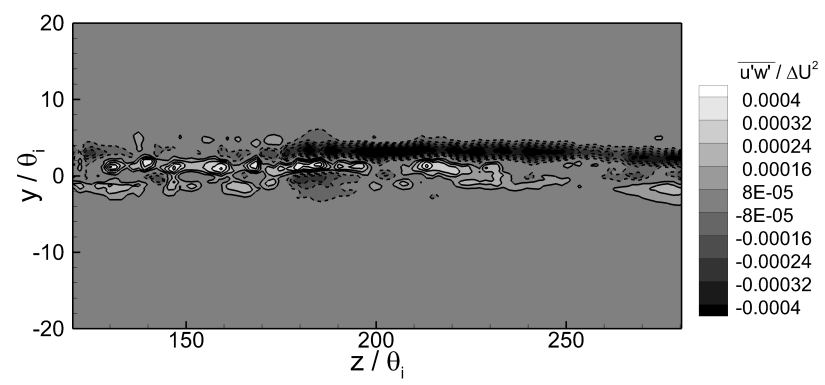

(a) Station 1.

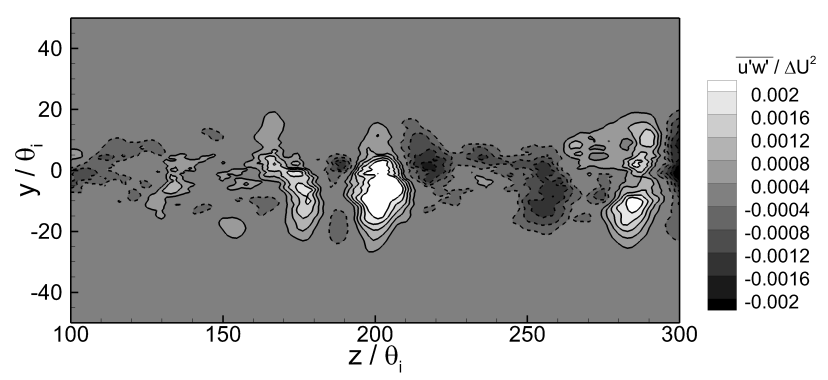

(b) Station 3.

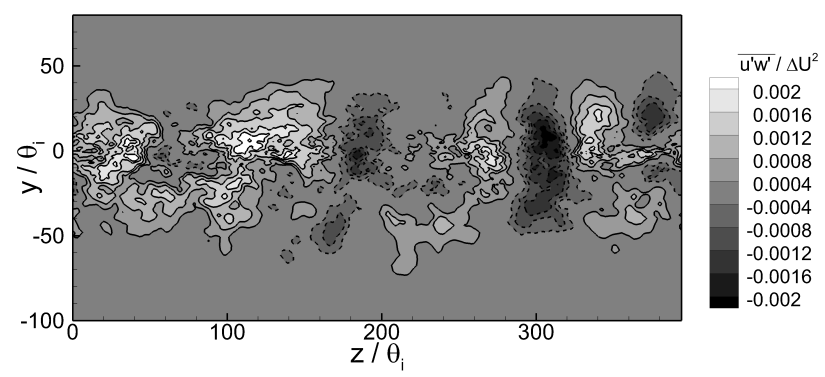

(c) Station 5 .

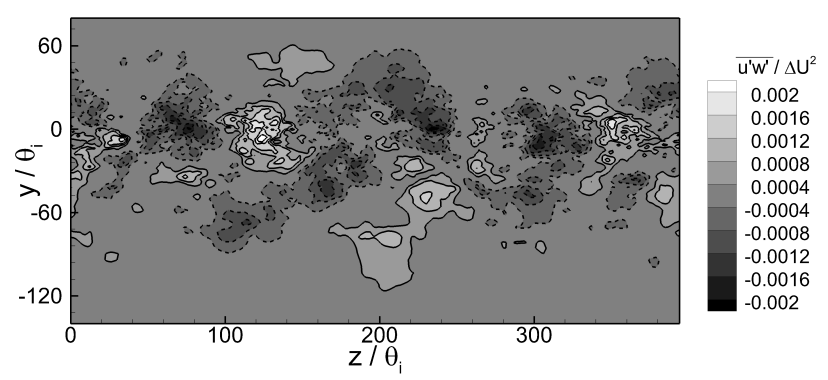

(d) Station 6 .

Figure 11: Cross-plane mean secondary shear stress contours in Case RRM-M. Note the changes in axes extent, and changes in contour levels, between images. 


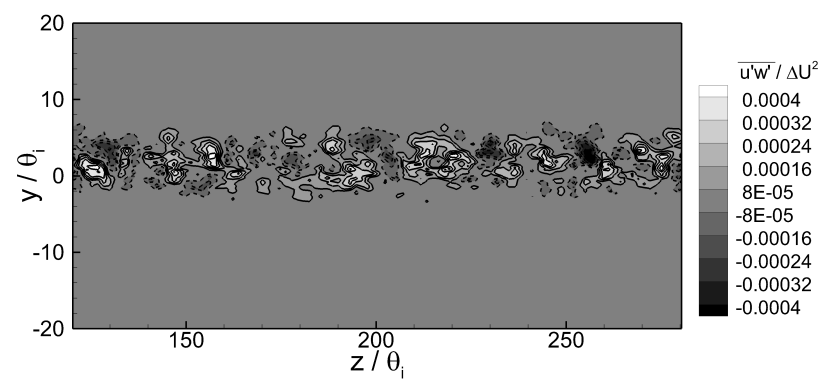

(a) Station 1.

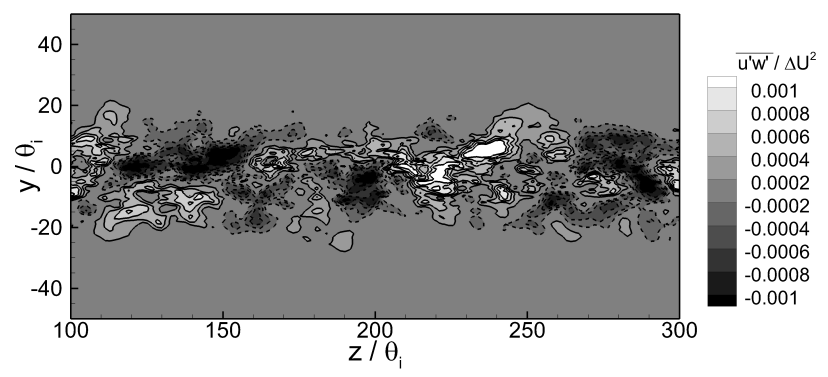

(b) Station 3.

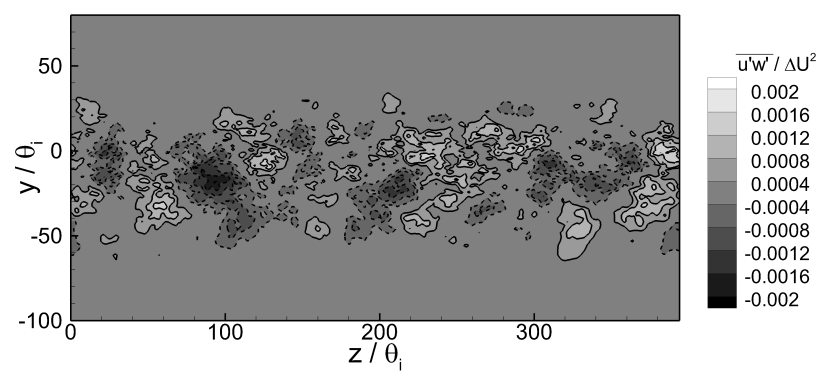

(c) Station 5 .

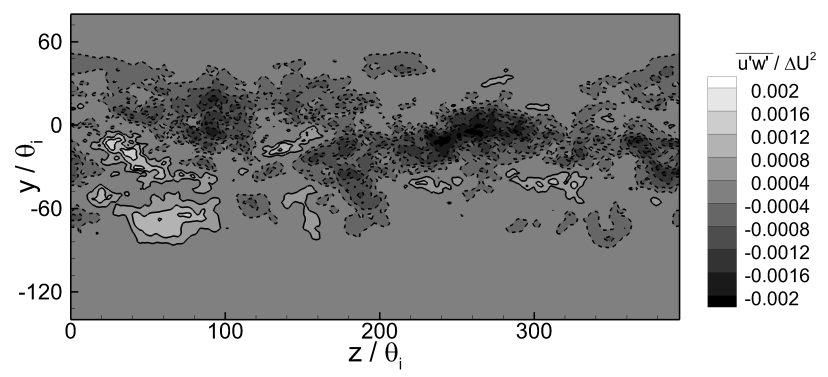

(d) Station 6 .

Figure 12: Cross-plane mean secondary shear stress contours in Case RRM-H. Note the changes in axes extent, and changes in contgur levels, between images. 


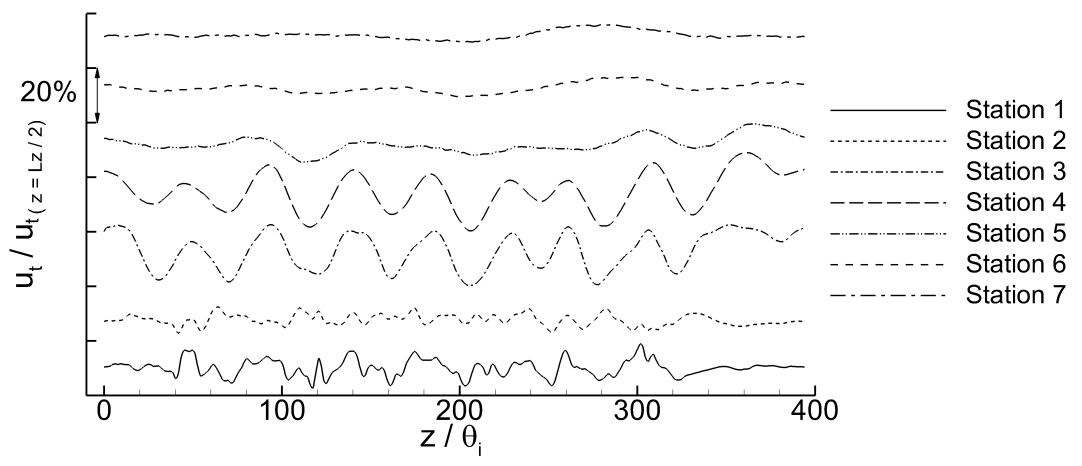

(a) RRM-L.

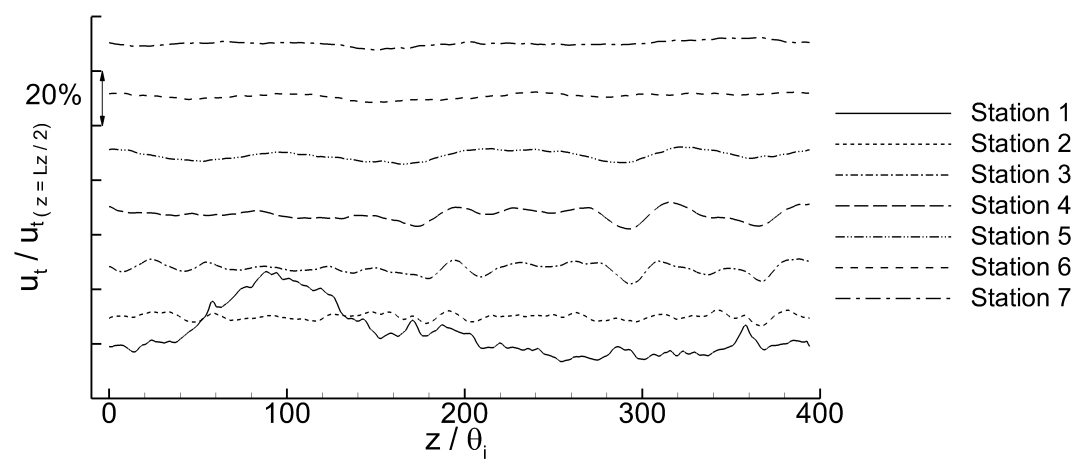

(b) RRM-M.

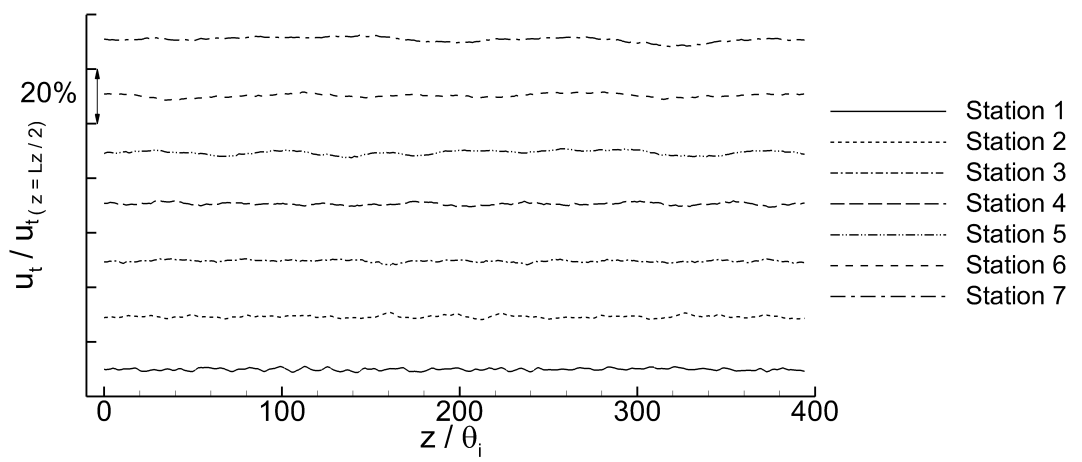

(c) RRM-H.

Figure 13: Spanwise variation of mean streamwise velocity in the plane of the splitter plate. 


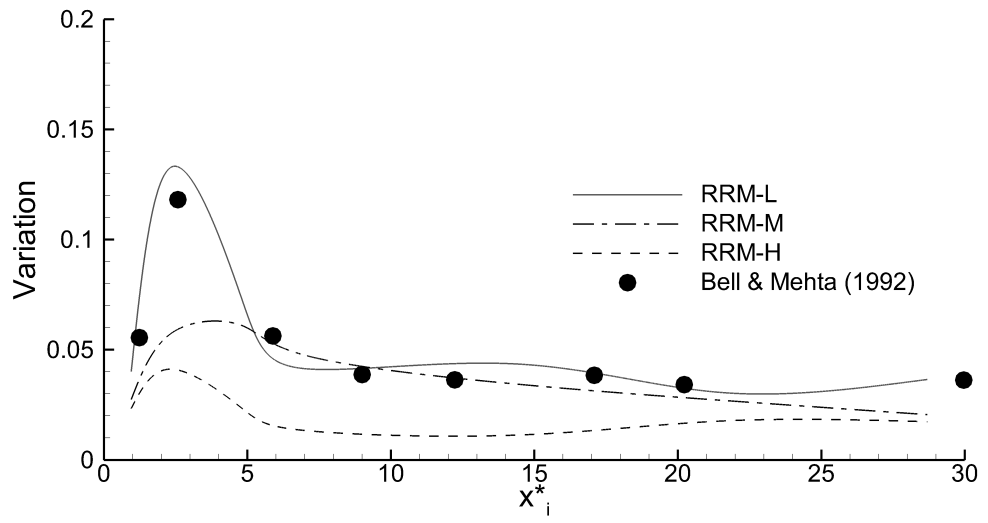

Figure 14: Standard deviation of mixing layer thickness. Comparable data of Bell \& Mehta [23] shown for reference. 